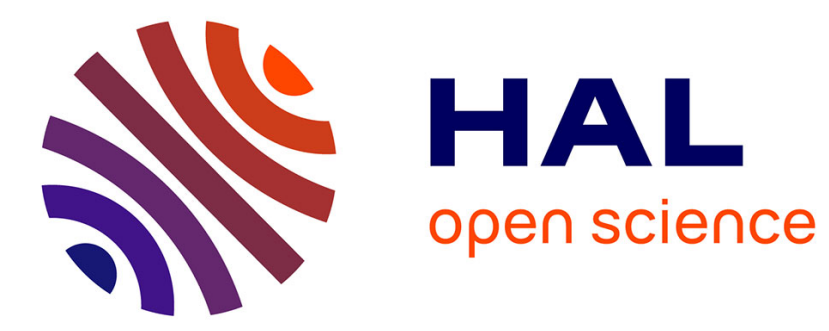

\title{
Optimal design of exchange networks with blind inputs and its application to Eco-industrial parks
}

David Salas Videla, Kien Cao Van, Didier Aussel, Ludovic Montastruc

\section{To cite this version:}

David Salas Videla, Kien Cao Van, Didier Aussel, Ludovic Montastruc. Optimal design of exchange networks with blind inputs and its application to Eco-industrial parks. Computers \& Chemical Engineering, 2020, 143, pp.107053. 10.1016/j.compchemeng.2020.107053 . hal-03080152

\section{HAL Id: hal-03080152 \\ https://hal.science/hal-03080152}

Submitted on 17 Dec 2020

HAL is a multi-disciplinary open access archive for the deposit and dissemination of scientific research documents, whether they are published or not. The documents may come from teaching and research institutions in France or abroad, or from public or private research centers.
L'archive ouverte pluridisciplinaire HAL, est destinée au dépôt et à la diffusion de documents scientifiques de niveau recherche, publiés ou non, émanant des établissements d'enseignement et de recherche français ou étrangers, des laboratoires publics ou privés. 


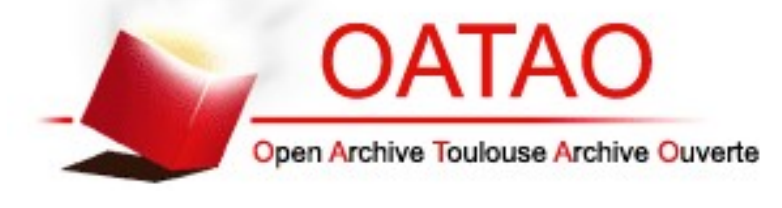

Open Archive Toulouse Archive Ouverte

OATAO is an open access repository that collects the work of Toulouse researchers and makes it freely available over the web where possible

This is an author's version published in: https://oatao.univ-toulouse.fr/27139

Official URL :

https://doi.org/10.1016/j.compchemeng.2020.107053

\section{To cite this version:}

Salas, David and Van, Kien Cao and Aussel, Didier and Montastruc, Ludovic Optimal design of exchange networks with blind inputs and its application to Eco-industrial parks. (2020) Computers \& Chemical Engineering, 143. 107053. ISSN 0098-1354

Any correspondence concerning this service should be sent to the repository administrator: tech-oatao@listes-diff.inp-toulouse.fr 


\title{
Optimal design of exchange networks with blind inputs and its application to Eco-industrial parks
}

\author{
David Salas ${ }^{\mathrm{a}, \mathrm{b}, \mathrm{c}, *}$, Kien Cao Van ${ }^{\mathrm{a}}$, Didier Aussel ${ }^{\mathrm{a}}$, Ludovic Montastruc ${ }^{\mathrm{b}}$ \\ a Laboratoire PROMES, UPR CNRS 8521, Université de Perpignan Via Domitia, Perpignan 66100, France \\ ${ }^{\mathrm{b}}$ Laboratoire de Génie Chimique, UMR 5503 CNRS/INP/UPS, Université de Toulouse, Toulouse 31432, France \\ ' Instituto de Ciencias de la Ingeniería, Universidad de O'Higgins, Libertador Bernardo O'Higgins 611, Rancagua, Chile
}

\section{A R T I C L E I N F O}

Keywords:

Optimization

Eco Industrial Park

Game theory

Single-Leader-Multi-Follower

\begin{abstract}
A B S T R A C T
Motivated by the design and optimization of the water exchange networks in Eco-Industrial Parks (EIP), we investigate the abstract Blind-Input model for general exchange networks. This abstract model is based on a Game Theory approach, formulating it as a Single-Leader-Multi-Follower (SLMF) game: at the upper level, there is an authority (leader) that aims to minimize the consumption of natural resources, while, at the lower level, agents (followers) try to minimize their operating costs. We introduce the notion of Blind-Input contract, which is an economic contract between the authority and the agents in order to ensure the participation of the latter ones in the exchange networks. More precisely, when participating in the exchange network, each agent accepts to have a blind input in the sense that she controls only her output fluxes, and the authority commits to guarantee a minimal relative improvement in comparison with the agent's stand-alone operation. The SLMF game is equivalently transformed into a single mixedinteger optimization problem. Thanks to this reformulation, examples of EIP of realistic size are then studied numerically.
\end{abstract}

\section{Introduction}

In the last few decades, the development of the industrialized countries has led to an increasing depletion of natural resources such as freshwater and energy (see, e.g., UNEP, 2000; Scientific and Organization), 2009). The conservation and sustainable use of such resources play an important role in both, environmental impact and business success within the industry. In response to preserve the environment while increasing the utilities of the enterprises, the concept of industrial ecology has emerged (Boix et al., 2015).

Industrial ecology (IE) was first introduced in Frosch and Gallopoulos (1989). They wrote "the consumption of energy and materials is optimized, waste generation is minimized and the effluents of one process ... serve as the raw material for another process". This is an approach to the industrial design of products and processes and the implementation of sustainable manufacturing strategies. The idea is directly related to another concept, industrial symbiosis, which involves "separate industries in a collective

\footnotetext{
* Corresponding author at: Laboratoire de Génie Chimique, UMR 5503 CNRS/INP/UPS, Université de Toulouse, 31432 Toulouse, France.

E-mail addresses: david.salas@uoh.cl (D. Salas), kien.van@promes.cnrs.fr (K.C. Van), aussel@univ-perp.fr (D. Aussel), ludovic.montastruc@ensiacet.fr (L. Montastruc).
}

approach to competitive advantage involving physical exchange of materials, energy, water and/or by-products" (see Chertow, 2000). One key concept of industrial symbiosis is then the exchange networks.

A perfect example of an exchange network which illustrates the notion of industrial symbiosis is the concept of Eco-Industrial Parks (EIP). This notion has several definitions, but one widely accepted is "an industrial system of planned materials and energy exchanges that seeks to minimize energy and raw materials use, minimize waste, and build sustainable economic, ecological and social relationships" Alexander et al. (2000); Boix et al. (2015); Montastruc et al. (2013).

Recently, in works of Boix et al. (2015) and Kastner et al. (2015), it has been pointed out that there is still a lack of systematic methods for designing the optimal configuration of an EIP. In previous studies (Boix et al., 2011; 2015; Montastruc et al., 2013), water integration networks (which is a classical example of EIP) were modeled as a cooperative economy, in the framework of multiobjective optimization (MOO). This approach consist in creating a vector function of $n+1$ coordinates given by

$C(F)=\left(\operatorname{Cost}_{1}(F), \ldots, \operatorname{Cost}_{n}(F), Z(F)\right)$

where $\operatorname{Cost}_{i}(\cdot)$ is the cost function of the enterprise $i, Z(\cdot)$ is the global consumption of natural resources, and $F$ is the flux 


\begin{tabular}{|c|c|}
\hline \multicolumn{2}{|c|}{ Nomenclature } \\
\hline \multicolumn{2}{|c|}{ Latin symbols } \\
\hline$n$ & number of independent agents \\
\hline$m$ & number of regulated agents \\
\hline $\mathcal{P}$ & set of independent agents \\
\hline $\mathcal{R}$ & set of regulated agents \\
\hline$I_{P}$ & index set of independent agents \\
\hline$I_{R}$ & index set of regulated agents \\
\hline$I$ & assembly of index sets $I_{P}$ and $I_{R}$ \\
\hline$I_{0}$ & assembly of index set $I$ and sink node 0 \\
\hline$E$ & network topology \\
\hline$E_{\max }$ & set of all admissible connections of the network \\
\hline$E^{c}$ & set of connections that are not in $E$ \\
\hline$E_{i, \mathrm{act}}$ & set of active arcs of agent $i$ \\
\hline$E_{s t}$ & stand-alone topology \\
\hline $\mathcal{E}$ & set of all valid topologies \\
\hline $\operatorname{Cost}_{i}(\cdot)$ & operating cost of agent $i$ \\
\hline $\mathrm{STC}_{i}$ & stand-alone cost of agent $i$ \\
\hline$C(i, j)$ & arc class of $(i, j)$ \\
\hline $\mathcal{C}_{i}$ & family of all arc classes exiting from agent $i$ \\
\hline$D$ & set of all arc classes of active agents \\
\hline$y$ & boolean variable \\
\hline$x_{i, j}$ & flux through the connection $(i, j)$ \\
\hline$x_{i}$ & outlet flux vector of agent $i$ \\
\hline$x_{-i}$ & vector of all fluxes not exiting from agent $i$ \\
\hline$x_{-i}^{P}$ & $\begin{array}{l}\text { vector of all fluxes exiting from an independent } \\
\text { agent other than } i\end{array}$ \\
\hline$x$ & complete vector of fluxes through the network \\
\hline$z_{i}$ & consumption of natural resource of the $i$ th agent \\
\hline$Z(\cdot)$ & total consumption of natural resources \\
\hline$g_{i}(\cdot)$ & input validation function of agent $i$ \\
\hline$F_{i}$ & $\begin{array}{l}\text { vector of fluxes exiting from enterprise } i \text { (water } \\
\text { exchange network) }\end{array}$ \\
\hline$F_{-i}$ & $\begin{array}{l}\text { vector of all fluxes not exiting from enterprise } i \\
\text { (water exchange network) }\end{array}$ \\
\hline$F^{P}$ & $\begin{array}{l}\text { vector of fluxes exiting from enterprises (water } \\
\text { exchange network) }\end{array}$ \\
\hline$F_{-i}^{P}$ & $\begin{array}{l}\text { vector of all fluxes exiting from an enterprise } \\
\text { other than } i \text { (water exchange network) }\end{array}$ \\
\hline$F^{R}$ & $\begin{array}{l}\text { vector of fluxes exiting from regeneration units } \\
\text { (water exchange network) }\end{array}$ \\
\hline$F$ & $\begin{array}{l}\text { flux vector describing the distribution in the wa- } \\
\text { ter exchange network }\end{array}$ \\
\hline$M_{i}$ & contaminant load of enterprise $i[\mathrm{~g} / \mathrm{h}]$ \\
\hline$C_{i, \mathrm{in}}, C_{i, \mathrm{out}}$ & $\begin{array}{l}\text { maximum contaminant concentration allowed in } \\
\text { inlet/outlet of processes [ppm] }\end{array}$ \\
\hline$C_{r, \text { in }}$ & $\begin{array}{l}\text { minimum inlet concentration allowed of reg. } \\
\text { units [ppm] }\end{array}$ \\
\hline$C_{r, \text { in }}$ & $\begin{array}{l}\text { exact outlet contaminant concentration of reg. } \\
\text { units [ppm] }\end{array}$ \\
\hline$A$ & the lifetime of the park [h] \\
\hline Coef & Penalization coefficient of stand-alone agents \\
\hline
\end{tabular}

\section{Acronyms}

EIP Eco-Industrial Park

GNEP generalized Nash equilibrium problem

Eq the set of equilibria for the induced GNEP

KKT Karush-Kuhn-Tucker

MILP Mixed-integer linear programming

MPEC mathematical programs with equilibrium constraint

SLMF Single-Leader-Multi-Follower

STC stand-alone cost

$$
\begin{array}{ll}
\begin{array}{l}
\text { Greek symbol } \\
\alpha
\end{array} & \text { the minimal relative gain that each agent ask for } \\
& \text { participating in the network } \\
c & \text { the marginal cost of freshwater consumption }[\$ / T] \\
\beta_{i, 0} & \text { the discharge cost of polluted water of enterprise } i \\
& {[\$ / T]} \\
\delta_{i, j} & \text { the cost sending polluted water from enterprise } i \text { to } \\
& j[\$ / T] \\
\Gamma_{r} & \text { the marginal cost of regenerating water }[\$ / T] \\
\psi & \text { power associated to } \Gamma_{r}
\end{array}
$$

vector describing the distribution in the exchange network. Then, the aim is to solve the problem of "minimizing" $C$ with respect to $F$, satisfying the physical constraints of the model. The result of such minimization is called a Pareto front, which consists in all vectors $F$ for which none of the coordinates of $C$ can be improved without worsen another one (McCain, 2010; Emmerich and Deutz, 2018). Usually an authority, representing the EIP's designer, selects one of this solutions considering as criteria the distance to an utopia point.

The main problem with such an approach is that points of the Pareto front are not necessarily economically stable: first, a Pareto point requires the enterprises to cooperate and share information, which is rarely the case of an EIP. Second, due to the noncooperative economy, the different enterprises may deviate from the selection of the authority since they may improve their cost function by unilaterally changing their operation. In terms of game theory, a solution of the MOO approach is a social optimization which may fail to respect incentives (see Nisan et al., 2007, Chapter 1).

To solve this incompatibility, again in the context of water integration networks, in the seminal work of Ramos et al. (2016), further developed in Ramos et al. (2018b), a novel game theory approach has been proposed, by modeling the EIP design problem as a Single-Leader-Multi-Follower (SLMF) game (see Aussel and Svensson, 2020; Hu and Fukushima, 2015): since the agents do not want to exchange information, a confidential centralization through an authority of the park is introduced. Then, at the upper level, there is the EIP authority which wants to minimize the consumption of natural resources $Z(F)$, while at the lower level, each enterprise tries to minimize her cost function $\operatorname{Cost}_{i}(F)$, related to her processes, consumption of natural resources and activity within the EIP. The authority of the park must choose the connections of the exchange network and the operation of the regeneration units, while each enterprise controls their consumption of natural resources and their output flux distribution. Based on the EIP authority decisions, all enterprises compete with each other in a parametric non-cooperative generalized Nash game with the strategies of the EIP authority as exogenous parameters. Fig. 1.1 shows the general scheme of such a model, where the enterprises are considered the economic agents of the game. We refer the reader to Nisan et al. (2007); Ichiishi (1983) for a primer in noncooperative games, to Pang and Fukushima (2005); Facchinei and Kanzow (2010) for a survey of Generalized Nash Equilibrium problems, and Dempe et al. (2015); Dempe and Zemkoho (2020) for the theory of bilevel optimization. For Single-Leader-Multi-Follower games, we refer to Hu and Fukushima (2015) and the references therein.

The main implicit assumption done in Ramos et al. (2016) is that each enterprise can only control her outlet distribution and her own fresh water consumption, but they are forced to accept whatever is sent to them through the exchange network. Furthermore, they have no knowledge about the particular actions of the other agents of the network, excepting only the amount and quality of the final inlet flux. In practice, this situation corresponds to the 


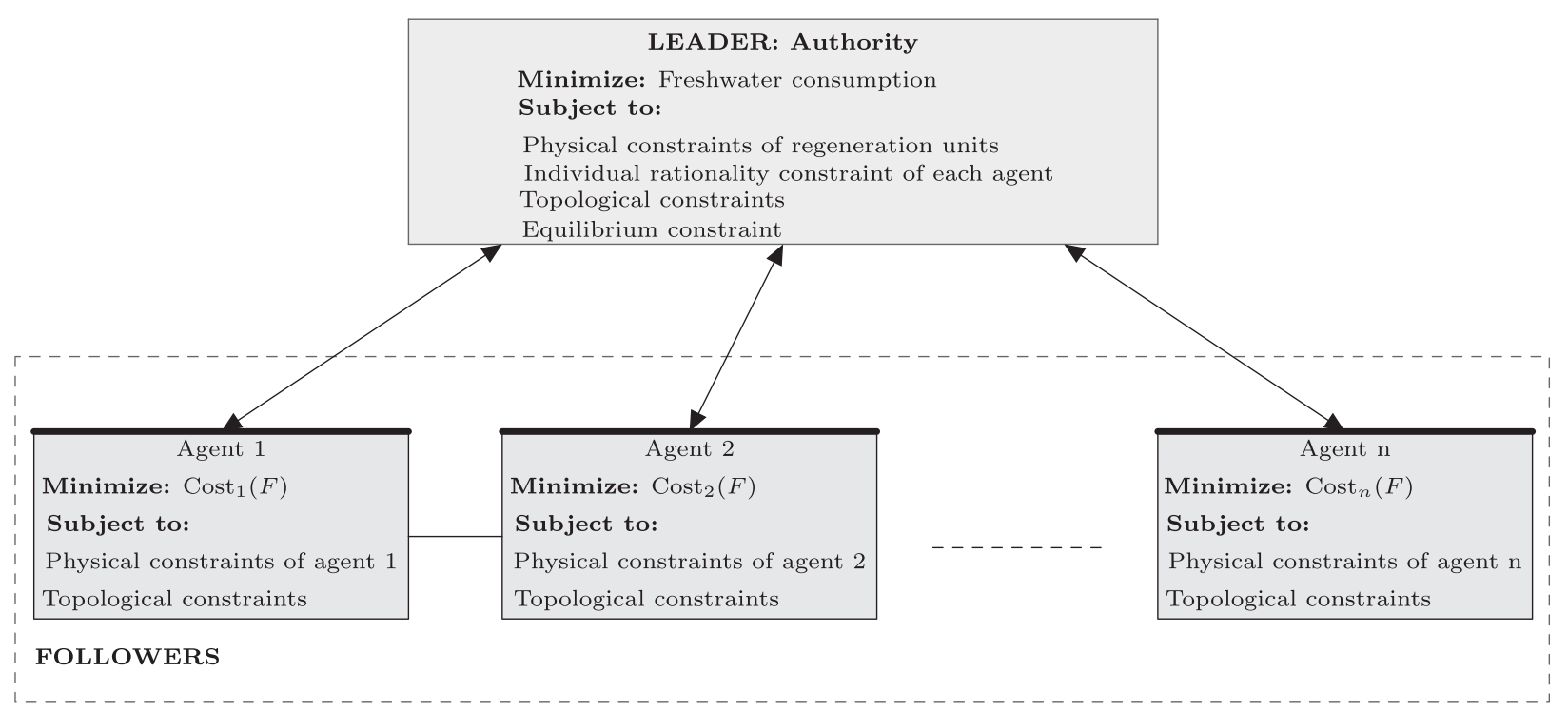

Fig. 1.1. General scheme of SLMF game.

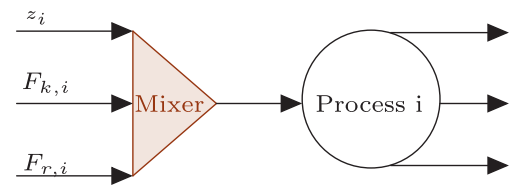

Fig. 1.2. Blind-Input Schema. $z_{i}, F_{k, i}$ and $F_{r, i}$ are freshwater consumption, wastewater sent from agent $k$ to $i$, and regenerated water sent from regeneration unit $r$ to $i$, respectively.

case when, at the entrance, each agent of the network has a mixer, and so she is only aware of the total input she is receiving, as Fig. 1.2 illustrates. In other words, when participating in the exchange network, each agent accepts to have a blind input.

While this model respects incentive consistency, it has two main drawbacks: the first one is that the rule that the park's authority imposes, that is, the blind input, is too restrictive. Indeed, under this paradigm, an enterprise may be forced to receive too much polluted water which could turn into higher costs than the stand-alone operation outside the park (examples are easy to construct with two enterprises). This violates the economical principle (well known in contract theory and mechanism design) of individual rationality: an enterprise will participate in the EIP only if it is convenient to her (see Jackson, 2014; Salanié, 2005; Bolton and Dewatripont, 2005); the second one is the strategy to compute a solution. In Ramos et al. (2016), the authors implemented the classic general approach to solve bilevel games, that is, to reformulate it as a mathematical programming with complementarity constraints (MPCC): loosely speaking, for a given network, they write the Karush-Kuhn-Tucker (KKT) conditions of each problem of the lower level game, and put them as constraints in the authority's problem. Then they implemented a Branch-and-Bound heuristic to obtain an approximated optimal exchange network, solving at each iteration the problem described above. However, it is known that the MPCC problems, which is a particular class of mathematical programming with equilibrium constraints (MPEC), are hard to solve (see, e.g., Baumrucker et al., 2008; Tseveendorj, 2013; Luo et al., 1996) and the heuristic itself doesn't guarantee a real solution of the problem (Aussel and Svensson, 2019; Dempe and Dutta, 2012). The literature on theoretical and algorithmic aspects of MPCC and MPEC problems is large and still an active field of research in mathematics.
In this work, we further investigate the model proposed in Ramos et al. (2016) for water exchange networks, briefly described in Section 2 and fully exposed in Section 5, but considering its abstract form for general exchange networks in Section 3.2. This abstract model is called Blind-Input model, since we consider the constraint of full acceptance for each enterprise. To solve the drawback given by the Individual Rationality constraint, we introduce in Section 3.3 the notion of Blind-Input contract, which is an economical contract between the authority and each enterprise in order to participate in the Blind-Input model. We prove that, under some linear structure of the costs functions $\operatorname{Cost}_{i}(\cdot)$ of each enterprise, the Blind-Input model can be reduced from a Single-Leader-MultiFollower problem to a single mixed-integer optimization problem. This reduction, which is our main contribution, is presented in Section 4.

The proposed reformulation of the Blind-Input model opens the door to a lot of new developments, from the numerical treatment of huge size problems thanks to classical MILP solvers to exhaustive search of equilibria for small/medium size applications. This is illustrated in the second part of the article for water exchange networks in Eco-Industrial Parks: Section 6 illustrates a case of study and the obtained results which are then discussed in Section 7. Conclusions and perspectives are presented in Section 8.

It is worth to mention that, even though this work is motivated by the design problem of water exchange networks, its abstract formulation presented in Section 3 allows to apply it to other type of networks, as for example energy networks (Boix et al., 2015; Neves et al., 2020). In Section 8, we will comment which are the main elements needed to apply the Blind-Input model to other contexts.

To survey our contributions, a comparison between this work and Ramos et al. (2016) is given in Table 1. It is important to mention that the nooncoperative approach using SLMF games in EIPs is very recent and, up to our knowledge, there is no other reference in the literature different from Ramos et al. (2016, 2018b) to compare our results with.

\section{Motivation: EIP model for water exchange}

In this section, we briefly describe the model of water exchange network used to describe Eco-Industrial Parks. The model can be found in Ramos et al. (2016); Boix et al. (2015) among others. A detailed version is further exposed in Section 5. 
Table 1

Comparison between Ramos et al. (2016) and the present work. The first two rows are related to the numerical examples used in each article.

\begin{tabular}{|c|c|c|}
\hline Comparison criteria & Ramos et al. (2016). & This work \\
\hline Number of enterprises & 3 & 15 \\
\hline $\begin{array}{l}\text { Number of processes per } \\
\text { enterprise }\end{array}$ & 5 & 1 \\
\hline Regeneration units & Yes & Yes \\
\hline $\begin{array}{l}\text { Admits multiple processes per } \\
\text { enterprise }\end{array}$ & Yes & No \\
\hline Tools to model the EIPs & SLMF game & SLMF game \\
\hline Presence of Blind-Input model & $\begin{array}{l}\text { Implicitly used. Not formalized. } \\
\text { Economic drawbacks. }\end{array}$ & $\begin{array}{l}\text { Explicit formalization. } \\
\text { Introduction of Blind-Input } \\
\text { contract as economic instrument. }\end{array}$ \\
\hline Solution Method & $\begin{array}{l}\text { MPCC reformulation + Branch- } \\
\text { and-Bound } \\
\text { Heuristic }\end{array}$ & $\begin{array}{l}\text { Mixed-Integer Linear } \\
\text { programming (MILP) reduction. }\end{array}$ \\
\hline Properties of the solution & $\begin{array}{l}\text { MPCC is hard to solve and existing } \\
\text { algorithms are not robust. The } \\
\text { solution of the MPCC may fail to } \\
\text { be a solution of the SLMF game. }\end{array}$ & $\begin{array}{l}\text { MILP alogirthms are robust. } \\
\text { Commercial solvers are available. } \\
\text { Any global solution of the MILP } \\
\text { problem is a global solution of the } \\
\text { SLMF game. }\end{array}$ \\
\hline $\begin{array}{l}\text { The operating cost of each } \\
\text { participating enterprise in the EIP } \\
\text { is lower than that of stand-alone. }\end{array}$ & No & Yes \\
\hline
\end{tabular}

In an Eco-Industrial Park (EIP), several enterprises exchange wastes to reduce the global consumption of natural resources. Each time an enterprise uses the natural resource in her industrial process, it comes out degraded, but still can be used as input for other enterprises in the park. One of the most classical examples of EIP (see, e.g., Boix et al., 2015; Boix et al., 2012 corresponds to the modeling of water exchange networks: each enterprise needs to consume water for her industrial processes and the outcoming water is partially polluted. Other examples using different natural resources like energy or heat can be found in Boix et al. (2011); Ramos et al. (2018a).

In Ramos et al. (2016), the design of a water exchange network is treated according to the following assumptions: first, the park has a fixed number of $n$ enterprises, each enterprise $i$ has to dilute an amount $M_{i}$ of contaminant, and the outlet concentration of contaminant must be less than a fixed concentration $C_{i, \text { out }}$. It is usually assumed that each enterprise $i$ has always an optimal operation, in the sense that the outlet concentration of contaminant is always equal to $C_{i, \text { out }}$.

Second, each enterprise $i$ can accept partially polluted water, but with a maximal concentration $C_{i, \text { in }}$. This concentration is measured after a mixer (see Fig. 1.2) in such a way that no enterprise can really know the operation of the other enterprises. However, this measurement, that we will denote $g_{i}$ and which depends on the actions of the other enterprises, allows enterprise $i$ to perform two fundamental actions: (1) report infeasibilities to the authority of the park, whenever the income water after the mixer doesn't fulfill the constraints; and (2) compute how much fresh water she needs to complete its process attaining the outlet concentration $C_{i, \text { out }}$.

Third, each enterprise has a cost function that depends on four factors: (1) the marginal cost of fresh water that she consumes, that we denote $c_{i}$; (2) the marginal cost of polluted water that she discharges to the environment, that we denote $\gamma_{i, 0}$; (3) the cost of sending polluted water through a connection of the park; and (4) the cost of receiving water from other agents of the park (other enterprises but also regeneration units controlled by the authority). The authority transfers the investment cost of the EIP to the enterprises via the last two costs: the first one, via a marginal cost $\gamma$ which depends on the connections that enterprise $i$ uses to send water; and the second one via a cost function $\operatorname{Cost}_{i}^{\text {in }}$ that will depend on the actions of the other enterprises.
Moreover, the main assumptions for the pricing instruments are that the prices of fresh water and discharged water are exogenous, and that the authority has no interest of making any profit, and therefore she will fix the prices of using the connections only to recover the investment and maintenance costs. This yields to the following scenario: each enterprise wants to minimize its cost of the use of water while the authority is in charge of the ecological concerns by minimizing the fresh water consumption.

Finally, as we mentioned before, the authority may have regeneration units. Each regeneration unit $r$ receives polluted water and reduces its contaminant concentration up to a certain value $C_{r \text {,out }}$. Then, it sends the water to the enterprises for reuse. The costs associated to the regeneration units are charged to the enterprises through the inlet cost function $\operatorname{Cost}_{i}^{\text {in }}$.

\section{Blind-input model}

Taking inspiration from the water management model described in Section 2, our aim in this section is to define the concept of abstract Blind-Input model for general exchange networks. We divided the model in two parts: the physical model, which gives the constraints that the network must satisfy; and the economical model, which gives the incentives of each agent of the network, as well as the Blind-Input contract between the agents and the authority, which will ensure the participation of the agents.

\subsection{Network model}

We first consider two main actors: a set of agents participating to an exchange network, and an authority that aims to minimize the consumption of natural resources. Among the agents, we differentiate a set $\mathcal{P}:=\left\{P_{1}, \ldots, P_{n}\right\}$ of independent agents, and a set $\mathcal{R}=\left\{R_{1}, \ldots, R_{m}\right\}$ of regulated agents (controlled by the authority). Regulated agents don't have economical motivations, but they act on the exchange network following the indications of the authority. In the context of water exchange in EIP, the independent agents are the enterprises, and the regulated ones model the regeneration units (Ramos et al., 2016).

We identify the independent agents with the index set $I_{P}=$ $\{1, \ldots, n\}$ and the regulated agents with $I_{R}=\{n+1, \ldots, n+m\}$. We set $I=I_{P} \cup I_{R}$ and $I_{0}=\{0\} \cup I$, where 0 represents the sink node.

We define an exchange network as a simple directed graph $\left(I_{0}\right.$, $E)$, where the edge $e=(i, j) \in E$ means that the agent $i$ can send 
part of her output to the agent $j$. The extra node 0 is identified as a sink node, which represent the possibility of discharge of the output. A valid network $\left(I_{0}, E\right)$ must satisfy the following five conditions:

I. $E \subseteq E_{\max }$, where $E_{\max }$ is the set of all admissible connections of the network.

II. $\left(I_{0}, E\right)$ is a simple graph, that is, there is no multiple edges nor graph loops in $E$.

III. Each independent agent $i \in I_{P}$ is connected with the sink node, that is, $(i, 0) \in E$.

IV. Each regulated agent $r \in I_{R}$ is not connected with the sink node, that is, $(r, 0) \notin E$.

$\mathrm{V}$. The sink node has not exit edges in $E$ that is $(0, i) \notin E_{\max }$, for any $i \in I$.

In what follows, we will call $E$ the topology of the network $\left(I_{0}\right.$, $E)$, and we will denote by $\mathcal{E}$ the set of all valid topologies. Nevertheless, in order to simplify notations, the network $\left(I_{0}, E\right)$ will be only represented by its topology $E$. Observe that this representation may lead to ambiguity, since the set $E$ doesn't allow to distinguish possible isolated regulated agents (independent agents are never isolated, given hypothesis III). However, this is not a problem, since any isolated regulated agent will be simply removed from the network.

For each edge $(i, j) \in E_{\max }$, we set the variable $x_{i, j}$ which represents the flux through the connection $(i, j)$. For each $i \in I$, we set $x_{i}:=\left(x_{i, j}:(i, j) \in E_{\max }\right)$, being thus the outcome vector of agent $i$. Finally, we set $x=\left(x_{i, j}:(i, j) \in E_{\max }\right)$, the complete vector of fluxes through the network.

To simplify the mathematical model we use, let us introduce some notation. We put $x^{R}:=\left(x_{r}: r \in I_{R}\right)$ and $x^{P}:=\left(x_{i}: i \in I_{P}\right)$. In what follows, for an agent $i \in I$, we will write

$x_{-i}:=\left(x_{k, j}:(k, j) \in E_{\max }, k \in I \backslash\{i\}\right)$,

$x_{-i}^{P}:=\left(x_{k, j}:(k, j) \in E_{\max }, k \in I_{P} \backslash\{i\}\right)$.

For a topology subset $A \subseteq E_{\max }$, we write

$\left.x\right|_{A}:=\left(x_{i, j}:(i, j) \in A\right)$.

Similarly, we define $\left.x_{i}\right|_{A},\left.x_{-i}\right|_{A},\left.x^{P}\right|_{A},\left.x_{-i}^{P}\right|_{A}$ and $\left.x^{R}\right|_{A}$. It will be useful also to denote $A^{c}:=E_{\max } \backslash A$.

\subsection{Physical model}

Let us fix a network topology $E \in \mathcal{E}$. If $E$ is implemented, then for each agent $i \in I$, the physical model of the network is given by the following six operational constraints:

1. Null fluxes outside the network: each agent can use only the connections in the topology $E$. Thus, we set

$\left.x_{i}\right|_{E^{c}}=0$,

that is, for every edge $(i, j) \notin E$, the flux $x_{i, j}$ is zero.

2. Consumption of natural resource: the consumption of natural resource of the $i$ th agent is given by the output fluxes of the other players, that is,

$z_{i}=z_{i}\left(x_{-i}\right)$.

This assumption is derived from an optimal response hypothesis: we assume that, for a given value of $x_{-i}$, the agent $i$ is capable of compute exactly the minimal amount of natural resource $z_{i}$ that she has to consume in order to perform her inner processes.

3. Balance constraint: the fluxes must satisfy the Kirchoff's law for the agent $i \in I$, that is,

$z_{i}\left(x_{-i}\right)+\sum_{(k, i) \in E} x_{k, i}=\sum_{(i, j) \in E} x_{i, j}$
Since 0 is the sink node, it is not subject to this balance constraint.

4. Input consistency: there exists a real-valued function $g_{i}$ which allows the agent $i \in I$ to validate the input coming from the other agents. We write this validation as an abstract inequality constraint

$g_{i}\left(x_{-i}\right) \leq 0$.

This constraint may represent maximal inlet fluxes, maximal inlet contaminant concentration, minimal inlet temperature, etc.

5. Positivity of fluxes: we assume that the fluxes on the graph, as well as the consumed natural resource are all positive, that is,

$x_{i} \geq 0 \quad$ and $\quad z_{i}\left(x_{-i}\right) \geq 0$.

6. Extra authority constraints: the exchange network may require additional constraints. We will model them here through an abstract inclusion

$x \in X$

where $X \subset \mathbb{R}^{\left|E_{\max }\right|}$ represents the abstract additional feasible set.

Remark 3.1. Here, we assume that the degradation of the natural resource is implicit in the connections of the topology $E$. In this general model, we suppose that agent $i$ can compute the degradation of its inlet flux through the functions $g_{i}$ and $z_{i}$.

An important element of this model is the total lack of direct information among the agents. We suppose that agent $i$ cannot know the actions of other agents, that is, she doesn't have access to the exact value of $x_{-i}$. However, she counts with indirect observations: even though $x_{-i}$ is unknown, the values of $z_{i}\left(x_{-i}\right)$, $g_{i}\left(x_{-i}\right)$ and the total inlet flux $\Sigma_{(k, i) \in E} x_{k, i}$ are available. For water exchange, this could be interpreted as a measurement of the amount of water and contaminant concentration after the mixer of Fig. 1.2. This is a very important feature of our model, since enterprises want to keep as much private information as possible. The only agent that has all information is the authority, who has access to the full vector $x$.

\subsection{Economical model}

In this setting, the network authority has two vectors of decision variables: she must choose the topology of the network $E \in \mathcal{E}$ and she controls the operation of the regulated agents, that is, the output vectors $x_{r}$, for every $r \in I_{R}$. Each independent agent $i \in I_{P}$ controls her output vector $x_{i}$.

We assume that the authority doesn't pay any cost associated to the implementation and operation of the network. Instead, she transfers all these costs through a function $\gamma: E_{\max } \rightarrow \mathbb{R}_{+}$, where $\gamma((i, j))=\gamma_{i, j}$ represents the marginal cost for sending one unit of flux through the connection $(i, j)$. Using this pricing, the independent agents will pay the investment cost of the network and also the operation of the regulated agents. Thus, if there is a connection $\left(r_{1}, r_{2}\right) \in E_{\max }$ between two regulated agents $r_{1}, r_{2} \in I_{R}$, we assume that $\gamma_{r_{1}, r_{2}}=0$.

Since all the investment cost is transfered to the independent agents, the authority is only concerned about minimizing the consumption of the natural resources, and so she aims to minimize the function

$Z(x):=\sum_{i \in I} z_{i}\left(x_{-i}\right)$

Remark 3.2. It could be argued that the authority must be also concerned about efficiency of the network, by considering the total investment cost of the park. However, we assume that the pricing 
instrument $\gamma$ is given exclusively to pay the investment and maintenance cost of the park, and that it will be implemented as efficiently as possible. The discussion over efficiency and right pricing instruments, is out of the scope of this work.

On the other hand any independent agent $i \in I_{P}$ wants to minimize her global cost $\operatorname{Cost}_{i}$, which can be separated into three components: the consumption of the natural resource $z_{i}\left(x_{-i}\right)$, the cost of discharging (using the connection $(i, 0)$ ), and the use of the exchange network. Therefore her cost function $\operatorname{Cost}_{i}$ is given as:

$\operatorname{Cost}_{i}\left(x_{i}, x_{-i}^{P}, x^{R}, E\right)=c_{i} \cdot z_{i}\left(x_{-i}\right)+\operatorname{Cost}_{i}^{\mathrm{in}}\left(x_{-i}^{P}, x^{R}\right)+\sum_{(i, j) \in E} \gamma_{i, j} \cdot x_{i, j}$.

where $\operatorname{Cost}_{i}^{\mathrm{in}}\left(x_{-i}^{P}, x^{R}\right)$ is the inlet operating cost of an agent $i$, and it satisfies that

$$
\sum_{(k, i) \in E_{\max }} x_{k, i}=0 \Rightarrow \operatorname{Cost}_{i}^{\text {in }}\left(x_{-i}^{P}, x^{R}\right)=0 .
$$

Observe that, the cost concerning the exit connections is linear, and so, the cost function is linear in the first component $x_{i}$.

Remark 3.3. Again, in terms of costs, agent $i$ doesn't have direct access to the actions of the other agents. However, she must pay an operating cost $\operatorname{Cost}_{i}^{\mathrm{in}}\left(x_{-i}^{P}, x^{R}\right)$ that is communicated to her by the authority. The choice of this function as pricing instrument could be studied, but this is out of the scope of the work. For now, we will suppose that agent $i$ has enough indirect information (through measurements after the mixer of Fig. 1.2) to consider the cost $\operatorname{Cost}_{i}^{\text {in }}\left(x_{-i}^{P}, x^{R}\right)$ as correct and therefore to accept it.

With this model, the minimization problem of the $i$ th independent agent (parametrized by the topology $E$, the actions of regulated agents $x^{R}$ and the actions of the other independent agents $x_{-i}^{P}$ ) leads to problem $P_{i}\left(x_{-i}^{P}, x^{R}, E\right)$ :

$$
\begin{aligned}
& \min _{x_{i}} \operatorname{Cost}_{i}\left(x_{i}, x_{-i}^{P}, x^{R}, E\right) \\
& \text { s.t. }\left\{\begin{array}{l}
z_{i}\left(x_{-i}\right)+\sum_{(k, i) \in E} x_{k, i}=\sum_{(i, j) \in E} x_{i, j} \\
g_{i}\left(x_{-i}\right) \leq 0 \\
z_{i}\left(x_{-i}\right) \geq 0 \\
x_{i} \geq 0 \\
\left.x_{i}\right|_{E^{c}}=0 .
\end{array}\right.
\end{aligned}
$$

We denote by $\operatorname{Eq}\left(x^{R}, E\right)$ the set of equilibria for the induced generalized Nash equilibrium problem (GNEP, for short) given by the vector $x^{R}$ and the topology $E$, that is

$$
x^{P} \in \mathrm{Eq}\left(x^{R}, E\right) \Longleftrightarrow \forall i \in I_{P}, x_{i} \text { solves } P_{i}\left(x_{-i}^{P}, x^{R}, E\right) \text {. }
$$

As we already discussed in Section 1 , the main problem of this model is that each independent agent only controls her output vector $x_{i}$, which is not realistic. She is forced by the authority to fully accept any inlet fluxes, which may be harmful. Thus, without any extra constraint, agent $i$ may not be willing to participate in the network.

Thus, to solve this problem, the authority must "buy" the participation of agent $i$. This is modeled by the Blind-Input contract: agent $i$ accepts to control only her output fluxes, and the authority commits to guarantee a minimal relative improvement of her cost, with respect to the stand-alone operation of agent $i$.

To formalize this requirement in the contract, let us denote the stand-alone topology by $E_{\text {st }} \in \mathcal{E}$, that is,

$E_{\mathrm{st}}:=\left\{(i, 0): i \in I_{P}\right\}$.

For each independent agent $i \in I_{P}$ we define the stand-alone cost $\mathrm{STC}_{i}$, as the optimal value of the problem $P_{i}\left(0,0, E_{\mathrm{st}}\right)$, that is,

$\mathrm{STC}_{i}=\left(c_{i}+\gamma_{i, 0}\right) \cdot z_{i}(0)$
In other words, $\mathrm{STC}_{i}$ is the cost of the $i$ th agent assuming that all other agents (independent and regulated) are inactive, i.e. when agent $i$ only send fluxes to the sink node and doesn't receive any complementary fluxes from other agents. Then, for each independent agent $P_{i}$, we can formulate the commitment of minimal improvement in the Blind-Input contract as the following constraint:

$\operatorname{Cost}_{i}\left(x_{i}, x_{-i}^{P}, x^{R}, E\right) \leq \alpha \cdot \mathrm{STC}_{i}$,

where $\alpha \in] 0,1[$ is the minimal relative gain that each agent ask for participating in the network. We assume that $\alpha>0$ since, it is impossible to eliminate all costs, and that $\alpha<1$ since no agent is indifferent concerning her participation in the network. Indeed, if $\operatorname{Cost}_{i}\left(x_{i}, x_{-i}^{P}, x^{R}, E\right)=\mathrm{STC}_{i}$, then the agent $i$ will prefer not to participate, since she has no gain, entering an exchange network is complicated and she knows she may be "helping the competition".

Finally, we can write the authority's problem as

$$
\text { s.t. } \begin{cases}\min _{E \in \mathcal{E}, x \in \mathbb{R}^{\left|E_{\max }\right|}} Z(x) \\ z_{r}\left(x_{-r}\right)+\sum_{(k, r) \in E} x_{k, r}=\sum_{(r, j) \in E} x_{r, j}, & \forall r \in I_{R}, \\ z_{r}\left(x_{-r}\right) \geq 0, & \forall r \in I_{R}, \\ g_{r}\left(x_{-r}\right) \leq 0, & \forall r \in I_{R}, \\ x^{R} \geq 0, & \\ \left.x^{R}\right|_{E^{c}}=0, & \\ x^{P} \in \mathrm{Eq}\left(x^{R}, E\right), & \\ \operatorname{Cost}_{i}\left(x_{i}, x_{-i}^{P}, x^{R}, E\right) \leq \alpha \cdot \mathrm{STC}_{i}, & \forall i \in I_{P} .\end{cases}
$$

The optimization problem (3.11) can be interpreted as follows: the authority will propose to the agents a topology $E$ and an operation $x \in \mathbb{R}^{\left|E_{\max }\right|}$ which satisfy all the physical constraints and also, such that the operation $x$ respects: 1) the incentive consistency, in the sense that no agent will have incentives to unilaterally deviate from the proposal due to the constraint $x^{P} \in \mathrm{Eq}\left(x^{R}, E\right)$; and 2) the individual rationality of each agent, in the sense that all agents will participate in the network since their participation has been bought through the constraint (3.10). The first criteria solves the economical inconsistency of MOO approach, and the second criteria solves the participation problem of the Single-Leader-Follower approach.

Remark 3.4. In this work, we do not claim novelty in the constraint $x^{P} \in \mathrm{Eq}\left(x^{R}, E\right)$. This is the main contribution of Ramos et al. (2016). However, the constraint (3.10) is new. In terms of modeling and in this context, the fact to "attract" the independent agents towards a participation in the general exchange network constitutes one of the important novelties of this work.

Remark 3.5. After reading the forthcoming Section 4, the reader will observe that all proofs and reductions could be made considering different values of $\alpha$ for each independent agent, that is, putting a value $\left.\alpha_{i} \in\right] 0$, 1[ for each $i \in I_{P}$. The value of $\alpha_{i}$ represents the "cost" of buying the participation of the $i$ th independent agent, which is exactly $\left(1-\alpha_{i}\right)$ STC $_{i}$. However, allowing to have different costs depending on the enterprise rises the natural question of how to decide these values. This problem lies in contract theory (for an introduction to the field, we refer to Bolton and Dewatripont (2005); Salanié (2005)) and it is out the scope of the article. Thus, we will consider only uniform values of $\alpha$, which can be interpreted as a public call for participation in the network. Uniform values of $\alpha$, however, imply that the cost of buying the participation of an agent is proportional to her size, due to the factor $\mathrm{STC}_{i}$.

Remark 3.6. An important factor we do not consider in this work is the rebound effect that costs reductions may have on the operation of agents. For example, it terms of water exchange, a diminu- 
tion of costs of agent $i$ with respect to $\mathrm{STC}_{i}$ may induce an increment of wastes production, that is, a variation in $M_{i}$. Thus, this rebound effect may change the value of $z_{i}\left(x_{-i}\right)$. Even though this is a very interesting problem, we suppose that the demand of natural resource is given by a fixed process, on which the costs within the network have no effect. In other words, the consumption of natural resource of each agent is inelastic.

\section{Mixed-integer programming reduction}

The formulation of the authority's problem (3.11) has the form of a general MPEC problem (see, e.g., Baumrucker et al., 2008; Tseveendorj, 2013; Luo et al., 1996). This section is devoted to prove that this MPEC formulation, which is known to be hard to solve, can be reformulated as a single Mixed-Integer programming problem.

This reduction can be interpreted as follows: Blind-Input models are a social optimization problem where, through Blind-Input contracts, the cooperation of each independent agent has been bought. This social optimization is also economically stable, since implicitly it respect an equilibrium constraint $\left(x^{P} \in \mathrm{Eq}\left(x^{R}, E\right)\right)$. This reduction/reformulation will be presented in three steps.

\subsection{Characterization of equilibria}

The following theorem characterizes the equilibrium set $\operatorname{Eq}\left(x^{R}\right.$, $E)$ as a system of equations. This allows to reduce the MPEC of problem (3.11) to a single optimization problem. The reduction we do here is based on the observation that, once every agent has committed to a Blind-Input contract, her actions become predictable through the cost functions. Thus, the authority can choose the network $E$ such that each action of an independent agent is induced to reach the social optimum.

To formalize this idea, let us introduce the notion of active arcs. Given a topology $E$, for each independent agent $i \in I_{P}$ we define the set of active arcs of $i$, denoted by $E_{i, \text { act }}$, as all the arcs $e \in E$ having minimum cost, that is,

$E_{i, \text { act }}:=\left\{(i, j) \in E: \gamma_{i, j}=\gamma_{i}^{*}:=\min _{(i, k) \in E} \gamma_{i, k}\right\}$.

As convention, for any regulated agent $r \in I_{R}$, we set $E_{r \text {,act }}=$ $\{(r, j):(r, j) \in E\}$.

Theorem 4.1. For $E \in \mathcal{E}$ and $x^{R} \geq 0$ fixed, the equilibrium set $\mathrm{Eq}\left(x^{R}\right.$, E) is given by

$$
\mathrm{Eq}\left(x^{R}, E\right)=\left\{\begin{array}{c}
z_{i}\left(x_{-i}\right)+\sum_{(k, i) \in E} x_{k, i}=\sum_{(i, j) \in E} x_{i, j} \\
g_{i}\left(x_{-i}\right) \leq 0 \\
x_{i}\left(x_{-i}\right) \geq 0 \\
\left.x_{i}\right|_{E_{i, a c t}^{c}}=0 \\
x_{i} \geq 0
\end{array}\right\}
$$

Thus, the authority's problem (3.11) is equivalent to the following Mixted-Integer Programming problem:

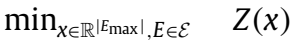

$$
\begin{aligned}
& \begin{cases}x \in X, & \\
z_{i}\left(x_{-i}\right)+\sum_{(k, i) \in E} x_{k, i}=\sum_{(i, j) \in E} x_{i, j}, & \forall i \in I \\
\left.x_{i}\right|_{E_{i, \text { act }}^{c}=0,} & \forall i \in I \\
g_{i}\left(x_{-i}\right) \leq 0, & \forall i \in I \\
z_{i}\left(x_{-i}\right) \geq 0, & \forall i \in I \\
\operatorname{Cost}_{i}\left(x_{i}, x_{-i}^{P}, x^{R}, E\right) \leq \alpha_{i} \cdot \mathrm{STC}_{i}, & \forall i \in I_{P} \\
x \geq 0 . & \end{cases}
\end{aligned}
$$

Proof. The second part of the proof is easily verified by replacing the constraint $x^{P} \in \mathrm{Eq}\left(x^{R}, E\right)$ by the system of equations in the right hand of equality (4.2), and then just reorganizing. Thus, we only need to prove (4.2).

To simplify notation, let us denote by $S\left(x^{R}, E\right)$ the right-hand set of (4.2). First, let us prove that $S\left(x^{R}, E\right) \subseteq \mathrm{Eq}\left(x^{R}, E\right)$. Fix $x^{P} \in S\left(x^{R}\right.$, $E$ ). Since $E_{i \text {,act }} \subset E$ for each $i \in I_{P}$, it is not hard to see that $x_{i}$ is a feasible set of $P_{i}\left(x_{-i}^{P}, x^{R}, E\right)$.

Now, fix $i \in I_{P}$ and let $x_{i}^{\prime}$ be another feasible point of $P_{i}\left(x_{-i}^{P}, x^{R}, E\right)$. Then, $x_{i}^{\prime} \geq 0$ and it satisfies the balance constraint (3.3), which yields that

$$
\begin{aligned}
\Delta \text { Cost }_{i} & =\sum_{(i, j) \in E} \gamma_{i, j} x_{i, j}^{\prime}-\gamma_{i}^{*}\left(\sum_{(i, j) \in E_{i, \mathrm{act}}} x_{i, j}\right) \\
& \geq \gamma_{i}^{*}\left(\sum_{(i, j) \in E} x_{i, j}^{\prime}-\sum_{(i, j) \in E_{i, \mathrm{act}}} x_{i, j}\right) \\
& \geq 0,
\end{aligned}
$$

where $\Delta \operatorname{Cost}_{i}:=\operatorname{Cost}_{i}\left(x_{i}^{\prime}, x_{-i}^{P}, x^{R}\right)-\operatorname{Cost}_{i}\left(x_{i}, x_{-i}^{P}, x^{R}\right)$ and the last inequality is due to the fact that

$$
\begin{aligned}
\sum_{(i, j) \in E} x_{i, j}^{\prime} & =z_{i}\left(x_{-i}\right)+\sum_{(k, i) \in E} x_{k, i} \\
& =\sum_{(i, j) \in E} x_{i, j}=\sum_{(i, j) \in E_{i, \text { act }}} x_{i, j} .
\end{aligned}
$$

Thus, $x_{i}$ solves $P_{i}\left(x_{-i}^{P}, x^{R}, E\right)$, and since this holds for every $i \in I_{P}$, we deduce that $x^{P} \in \mathrm{Eq}\left(x^{R}, E\right)$.

Now, let us prove that $\mathrm{Eq}\left(x^{R}, E\right) \subseteq S\left(x^{R}, E\right)$. Let $x^{P} \in \mathrm{Eq}\left(x^{R}, E\right)$, and suppose that $x^{P} \notin S\left(x^{R}, E\right)$. Since for each $i \in I_{P}$ the vector $x_{i}$ is a feasible point of $P\left(x_{-i}^{P}, x^{R}, E\right)$, the only way for $x^{P}$ not to belong to $S\left(x^{R}, E\right)$ is that there exist $i_{0} \in I_{P}$ such that $\left.x_{i_{0}}\right|_{E_{i_{0} \text { act }}^{c}} \neq 0$. Thus, there is $\left(i_{0}, j_{0}\right) \in E \backslash E_{i_{0} \text {,act }}$ such that $x_{i_{0}, j_{0}}>0$. Let $\left(i_{0}, j_{1}\right) \in E_{i, \text { act }}$ (which is nonempty by definition) and let us consider the vector $x_{i_{0}}^{\prime}$ given by

$x_{i_{0}, k}^{\prime}= \begin{cases}x_{i_{0}, k} & \text { if } k \in I \backslash\left\{j_{0}, j_{1}\right\}, \\ 0 & \text { if } k=j_{0}, \\ x_{i_{0}, j_{1}}+x_{i_{0}, j_{0}} & \text { if } k=j_{1} .\end{cases}$

We have that $x_{i_{0}}^{\prime} \geq 0$ (since $x_{i_{0}} \geq 0$ ) and also

$z_{i}\left(x_{-i_{0}}\right)+\sum_{\left(k, i_{0}\right) \in E} x_{k, i_{0}}=\sum_{\left(i_{0}, j\right) \in E} x_{i_{0}, j}=\sum_{\left(i_{0}, j\right) \in E} x_{i_{0}, j}^{\prime}$.

Thus, since $x_{-i_{0}}$ remains the same, $x_{i_{0}}^{\prime}$ is a feasible point of $P_{i}\left(x_{-i_{0}}^{P}, x^{R}, E\right)$. Furthermore, denoting by $\Delta$ Cost $_{i_{0}}=$ $\operatorname{Cost}_{i_{0}}\left(x_{i_{0}}^{\prime}, x_{-i_{0}}^{P}, x^{R}, E\right)-\operatorname{Cost}_{i_{0}}\left(x_{i_{0}}, x_{-i_{0}}^{P}, x^{R}, E\right)$, we have that

$$
\begin{aligned}
\Delta \operatorname{Cost}_{i_{0}} & =\sum_{\left(i_{0}, j\right) \in E} \gamma_{i_{0}, j} x_{i_{0}, j}^{\prime}-\sum_{\left(i_{0}, j\right) \in E} \gamma_{i_{0}, j} x_{i_{0}, j} \\
& =\left(\gamma_{i_{0}, j_{1}}-\gamma_{i_{0}, j_{0}}\right) x_{i_{0}, j_{0}} \\
& =\left(\gamma^{*}-\gamma_{i_{0}, j_{0}}\right) x_{i_{0}, j_{0}}<0,
\end{aligned}
$$

since, by construction, $\gamma_{i_{0}, j_{0}}>\gamma^{*}$. This yields that $x_{i_{0}}$ doesn't solve $P_{i}\left(x_{-i_{0}}^{P}, x^{R}, E\right)$, which is a contradiction. Thus, $x^{P} \in S\left(x^{R}, E\right)$, finishing the proof.

Intuitively, the above theorem says that, given a topology $E$, each independent agent $i \in I_{P}$ will only use the connections of minimal cost to send the excess of flux, that is, she will use only her active arcs. Furthermore, each independent agent is indifferent to the distribution of fluxes among the active arcs, so any feasible vector $x^{P}$ satisfying the constraint $\left.x_{i}\right|_{E_{i, \text { act }}^{c}}=0$ for every $i \in I_{P}$ must be an equilibrium. This simplification is strongly based on the linearity of the costs functions with respect to the agent's variable $x_{i}$. 


\subsection{Mixed-integer formulation}

Theorem 4.1 establishes the remarkable fact that the MPEC formulation of the authority's problem can be reformulated as a "classical" programming problem. But actually, a part of the variables of this programming problem lies in the set of topologies of the exchange network and so, it can be considered as difficult to implement numerically. This is why, in this section, we will show how one can finally work with a more classical mixed-integer programming problem.

Let us first introduce the key notion that we will use to arrive to the final formulation, that is, what we call arc classes: let $(i, j) \in E_{\max }$. We define the arc class of $(i, j)$ as the set

$C(i, j):=\left\{\begin{array}{ll}\left\{(i, k) \in E_{\max } \gamma_{i, k}=\gamma_{i, j}\right\} & \text { if } i \in I_{P} \\ \left\{(i, k) \in E_{\max }\right\} & \text { if } i \in I_{R}\end{array}\right.$.

We denote by $\mathcal{C}_{i}$ the family of all arc classes exiting from $i$, that is, $\mathcal{C}_{i}=\left\{C(i, j):(i, j) \in E_{\max }\right\}$. Finally, for $C \in \mathcal{C}_{i}$ we define the utilization cost of the class by

$\gamma(C):=\gamma_{i, j}$

where $(i, j)$ is any representative of $C$.

Observe that, for two $\operatorname{arcs}(i, j),(i, k) \in E_{\max }$ such that $\gamma_{i, j}=\gamma_{i, k}$, one has that $C(i, j)=C(i, k)$. Thus, a class $C \in \mathcal{C}_{i}$ may have many representations of the form $C(i, j)$. Furthermore, the family $\mathcal{C}_{i}$ induces a partition of the set of arcs "exiting from" agent $i$, that is

- $\bigcup_{C \in \mathcal{C}_{i}} C=\left\{e \in E_{\max }: e=(i, j)\right.$ for some $\left.j \in I_{0}\right\}$.

- For any two classes $C, C^{\prime} \in \mathcal{C}_{i}$, either $C=C^{\prime}$ or $C \cap C^{\prime}=\emptyset$.

Moreover, it is not hard to verify that for each topology $E \in \mathcal{E}$ and for each agent $i \in I_{P}$, there exists one class $C \in \mathcal{C}_{i}$ such that

$E_{i, \text { act }} \subseteq C$,

and this class must satisfy that

$\gamma(C) \leq \gamma(C(i, 0))$

This class is then given by $C=C(i, j)$ where $(i, j)$ is any element of $E_{i, \text { act }}$. We will call it the active class of $E$ of the agent $i$, and we will denote it by $C_{i}(E)$.

Without loss of generality, we will assume that every class $C \in \mathcal{C}_{i}$ satisfies (4.6). If not, any connection in a class violating (4.6) would never been used, and therefore, in practice, they can be erased from $E_{\max }$ without changing the problem.

Now, let $D=\bigcup_{i \in I_{P}} \mathcal{C}_{i}$, the set of all arc classes of independent agents. We introduce the boolean variable $y=\left(y_{C}\right)_{C \in D} \in\{0,1\}^{|D|}$ in the following way: for each independent agent $i \in I_{P}$ and each arc class $C \in \mathcal{C}_{i}$, we set

$y_{C}= \begin{cases}1 & \text { if } C \text { is the active class of } i, \\ 0 & \text { otherwise. }\end{cases}$

From $y \in\{0,1\}^{|D|}$, we will build the graph associated to $y$ as

$E(y)=\left(\bigcup\left\{: y_{C}=1\right\}\right)$

$\left.\cup\{, 0): i \in I_{P}\right\} \cup\left\{(r, j) \in E_{\max }: r \in I_{R}\right\}$.
We consider then the following Mixed-Integer optimization problem:

$$
\begin{aligned}
& \min _{x \in \mathbb{R}^{N}, y \in\{0,1\}|D|} Z(x) \\
& \text { s.t. } \begin{cases}x \in X, & \\
z_{i}\left(x_{-i}\right)+\sum_{(k, i) \in E_{\max }} x_{k, i}=\sum_{(i, j) \in E_{\max }} x_{i, j}, & \forall i \in I, \\
\sum_{C \in \mathcal{C}_{i}} y_{C}=1, & \forall i \in I_{P}, \\
\sum_{(i, j) \in C} x_{i, j} \leq B \cdot y_{C}, & \forall C \in D, \\
g_{i}\left(x_{-i}\right) \leq 0, & \forall i \in I_{P}, \\
z_{i}\left(x_{-i}\right) \geq 0, & \forall i \in I, \\
\operatorname{Cost}_{i}\left(x_{i}, x_{-i}^{P}, x^{R}, E(y)\right) \leq \alpha_{i} \cdot \operatorname{STC}_{i}, & \forall i \in I_{P}, \\
x \geq 0, & \end{cases}
\end{aligned}
$$

where $B$ is a real number chosen arbitrarily, but bigger than the maximum of the total entering flux over all enterprises. A simple option to set $B$ is the value $Z(0)$, which corresponds to the total consumption of the natural resource when there is no exchange network.

Here, the constraint $\sum_{C_{\in} \mathcal{C}_{i}} y_{C}=1$ says that, for the $i$ th agent, only one class is active. Also, the constraint

$\sum_{(i, j) \in C} x_{i, j} \leq B \cdot y_{C}, \quad \forall C \in D$

ensures that, whenever $(i, j)$ doesn't belong to the active class of the $i$ th agent, then $x_{i, j}=0$.

Theorem 4.2. For every feasible point $(x, y)$ of (4.8), the pair $(x, E(y))$ is a feasible point of (4.3). Conversely, for every feasible point $(x, E)$ of (4.3), the pair $\left(x, y^{E}\right)$ is a feasible point of (4.8), where $y^{E} \in\{0,1\}^{|D|}$ is given by

$y_{C}^{E}=\left\{\begin{array}{lc}1 & \text { if } C=C_{i}(E) \text { for some } i \in I_{P}, \\ 0 & \text { otherwise. }\end{array}\right.$

Finally, one has that

1. if $(x, E)$ is an optimal solution of (4.3), then $\left(x, y^{E}\right)$ is an optimal solution of (4.8).

2. if $(x, y)$ is an optimal solution of (4.8), then $(x, E(y))$ is an optimal solution of (4.3).

Proof. Let $(x, y)$ be a feasible point of (4.8). Let us fix an agent $i \in I_{P}$ and let $C_{i}$ be the unique class in $\mathcal{C}_{i}$ such that $y_{C_{i}}=1$. Then, by construction, we know that

$$
E(y)_{i, \text { act }}=C_{i} \text { and } \sum_{(i, j) \in E_{\max } \backslash C_{i}} x_{i, j} \leq B \cdot \sum_{C \in \mathcal{C}_{i} \backslash\left\{C_{i}\right\}} y_{C}=0 \text {. }
$$

We deduce then that

$\left.x_{i}\right|_{E(y)_{i, \text { act }}^{c}}=0$.

Since this constraint is valid for every active agent $i \in I_{P}$, and since $E(y)$ contains all exiting arcs for every regulated agent $r \in I_{R}$, we can rewrite the balance constraint in problem (4.8) as

$z\left(x_{-i}\right)+\sum_{(k, i) \in E(y)} x_{k, i}=\sum_{(i, j) \in E(y)} x_{i, j}, \forall i \in I$.

We deduce then that $(x, E(y))$ is a feasible point of problem (4.3). Now, let $(x, E)$ be a feasible point of problem (4.3). By inclusion (4.5), for each independent agent $i \in I_{P}$, there exists a unique active class $C_{i}(E)$. Let us define $y^{E} \in\{0,1\}^{|D|}$ as in the statement of the theorem.

Then, for every $i \in I_{P}, \sum_{C \in \mathcal{C}_{i}} y_{C}^{E}=1$. Now, fix a class $C \in D$, and let $i \in I_{P}$ such that $C \in \mathcal{C}_{i}$. We have that

$\sum_{(i, j) \in C} x_{i, j} \leq \begin{cases}B=B \cdot y_{C}^{E} & \text { if } C=C_{i}(E), \\ 0=B \cdot y_{C}^{E} & \text { if } C \neq C_{i}(E),\end{cases}$ 
where the second inequality comes from the fact that, whenever $C \neq C_{i}(E)$, then $C \subseteq E_{i, \text { act }}^{c}$ and so $\left.x_{i}\right|_{C}=0$. that

$\operatorname{Cost}_{i}\left(x_{i}, x_{-i}^{P}, x^{R}, E\left(y^{E}\right)\right)=\operatorname{Cost}_{i}\left(x_{i}, x_{-i}^{P}, x^{R}, E\right)$,

and so, the constraint (3.10) is satisfied. We deduce that $\left(x, y^{E}\right)$ is a feasible point of $(4.8)$, since all other constraints are directly satisfied given that $(x, E)$ is feasible for problem (4.3).

Now, let us assume that $(x, E)$ is also optimal for problem (4.8). From the development above, for every other feasible point $\left(x^{\prime}, y^{\prime}\right)$ of (4.8), we know that $\left(x^{\prime}, E\left(y^{\prime}\right)\right)$ is also a feasible point of problem (4.3), and so, $Z(x) \leq Z\left(x^{\prime}\right)$. Thus, $\left(x, y^{E}\right)$ is optimal for the problem (4.8).

Let now assume that $(x, y)$ is an optimal solution of problem (4.8) and suppose, by absurd, that $(x, E(y))$ is not optimal for problem (4.3). Then, there exists a feasible point $\left(x^{\prime}, E^{\prime}\right)$ of problem (4.3) such that $Z\left(x^{\prime}\right)<Z(x)$. But, as proved above, $\left(x^{\prime}, y^{E^{\prime}}\right)$ is also feasible for problem (4.8), showing that $(x, y)$ is not optimal for (4.8), which is a contradiction. The proof is then completed.

The reader could observe that, a priori, the mixed-integer problem (4.8) is smaller than problem (4.3) in some sense, since it admits only certain topologies (those ones of the form $E(y)$ for some feasible point $y \in\{0,1\}^{|D|}$ ). However the above theorem shows that the set of flux distributions $x$ for which $(x, E)$ is an optimal solution of (4.3) for at least one topology $E$ coincides with the set of flux distributions $x$ for which $(x, y)$ is an optimal solution of (4.8) for at least one $y$.

\subsection{Null class as exit option}

Physically, we know that the network has always a feasible point, which is the stand-alone configuration, that is, the topology $E_{\text {st }}$ and the fluxes given by the individual operations of the independent agents and inactivity of the regulated ones. However, when we include the individual rationality constraint (3.10), the problem may become infeasible.

Infeasibility of problem (4.3) means that the authority is not capable to find a solution that respect the Blind-Input contracts with all the agents. Thus, we need to include the possibility of excluding some agents from the network.

Formally, for each independent agent $i \in I_{P}$, we include a boolean variable $y_{i, \text { null }} \in\{0,1\}$ such that

$y_{i, \text { null }}= \begin{cases}1 & \text { if } i \text { breaks the Blind-Input contract, } \\ 0 & \text { otherwise. }\end{cases}$

With this new variable, we modify problem (4.8) as follows:

1. For each agent $i \in I_{P}$, we put

$$
y_{i, \text { null }}+\sum_{C \in \mathcal{C}_{i}} y_{C}=1,
$$

meaning that, either one arc class is active or the agent is outside the network.

2. For each agent $i \in I_{P}$, we put

$$
\begin{gathered}
\sum_{(i, j) \in C(i, 0)} x_{i, j} \leq B \cdot\left(y_{C(i, 0)}+y_{i, \text { null }}\right) \\
\sum_{(i, j) \in E_{\max }, j \neq 0} x_{i, j} \leq B \cdot\left(1-y_{i, \text { null }}\right)
\end{gathered}
$$

This is to ensure that, if the agent breaks the Blind-Input contract, then she will use the discharge arc $(i, 0)$.

3. For each agent $i \in I_{P}$, we put

$$
\sum_{(k, i) \in E_{\max }} x_{k, i} \leq B \cdot\left(1-y_{i, \text { null }}\right) \text {. }
$$

This constraint establishes that, if the agent breaks the BlindInput contract, then nobody can send her any flux.

4. For each agent $i \in I_{P}$, we put

$$
\operatorname{Cost}_{i}\left(x_{i}, x_{-i}^{P}, x^{R}, E(y)\right) \leq \alpha_{i} \mathrm{STC}_{i} \cdot\left(1-y_{i, \text { null }}\right)+\mathrm{STC}_{i} \cdot y_{i, \text { null }} .
$$

Here, the individual rationality constraint is active only when $y_{i, \text { null }}=0$. Otherwise, since the agent is not connected to the network, her cost will coincide with $\mathrm{STC}_{i}$.

We set $\bar{D}=D \cup\left\{\right.$ Null $\left._{i}: i \in I_{P}\right\}$, where Null $_{i}$ is the null class, associated to $y_{i, \text { null }}$, and $D_{0}=D \backslash\left\{C(i, 0): i \in I_{P}\right\}$. Denoting

$\operatorname{STC}_{i}\left(y_{i, \text { null }}\right):=\alpha_{i} \mathrm{STC}_{i} \cdot\left(1-y_{i, \text { null }}\right)+\operatorname{STC}_{i} \cdot y_{i, \text { null }}$,

the new optimization problem becomes

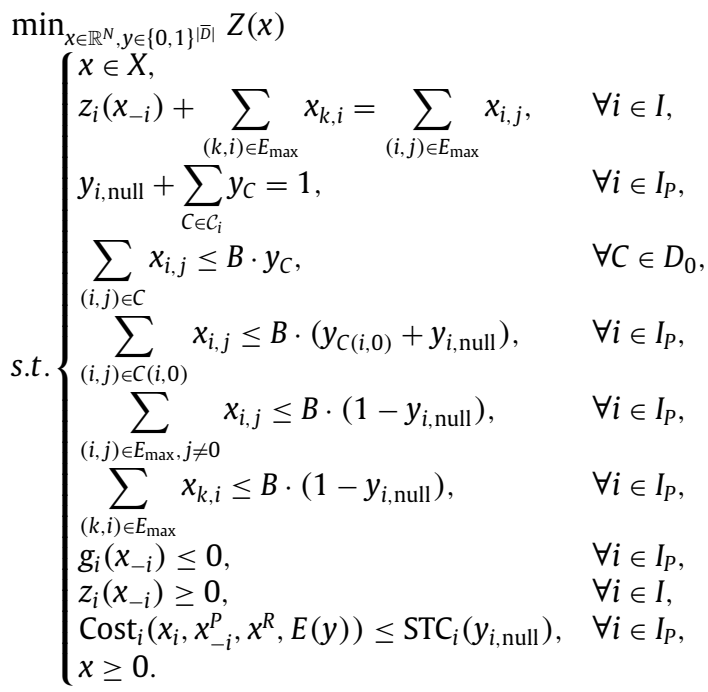

Observe that, whenever $y_{i, \text { null }}=0$, then all constraints for the $i$ th agent are the same that those established in problem (4.8). Also, if $y_{i \text {,null }}=1$, the only feasible solution for $i$ is the stand-alone operation. Thus, in this new problem, the authority first choose all the agents that will participate in the network, represented by the set

$I_{P}^{\prime}=\left\{i \in I_{P}: y_{i, \text { null }}=0\right\}$,

and then it solves problem (4.8) replacing $I$ by $I^{\prime}=I_{P}^{\prime} \cup I_{R}$. Of course, as it is formulated, the authority takes both decisions simultaneously, by solving problem (4.10). It is not hard to verify that any optimal solution of problem (4.10) is an optimal solution of Problem (4.8) for the reduced set of agents $I^{\prime}$. We leave this verification to the reader.

\section{Blind-Input model for water exchange networks}

In this section we come back to our original motivation presented in Section 2, the water exchange networks in Eco-Industrial Parks. We are now ready to describe in detail the model, and how it fits into the Blind-Input model developed so far.

First, an EIP consists in a set of enterprises $\mathcal{P}:=\left\{P_{1}, \ldots, P_{n}\right\}$, that are connected in an exchange network. Each enterprise $P_{i}$ can be connected either to other enterprises, or to some regeneration units, which we denote $\mathcal{R}:=\left\{R_{1}, \ldots, R_{m}\right\}$. The regeneration units are controlled by a central authority. This authority plays the role of designer (when deciding the connections within the network) and regulator of the park's operation. Finally, we include a sink node 0 , that represents a wastes' pit to discharge useless polluted water. We identify $\mathcal{P}$ with the index set $I_{P}:=\{1, \ldots, n\}$ and $\mathcal{R}$ with $I_{R}:=\{n+1, \ldots, n+m\}$. We put $I=I_{P} \cup I_{R}$ and $I_{0}=\{0\} \cup I$. Finally, 
for an agent of the park we refer either to an enterprise or to a regeneration unit.

Each enterprise $i \in I_{P}$ generates a fixed amount of pollutant $M_{i}$ [g], coming from her internal production process, that needs to be diluted before exiting the enterprise. To do so, enterprise $i$ must buy an amount of fresh water $z_{i}[\mathrm{~T} / \mathrm{h}]$ such that, after dilution, the pollutant concentration in the exit flux is less than a limit concentration $C_{i, \text { out }}[\mathrm{ppm}]$. If the enterprise discharges this polluted water into the sink node, she has to pay a tax associated to the contamination she is producing. We will assume a hypothesis of optimal response: each enterprise $i \in I_{P}$ consumes exactly the fresh water she needs to attain $C_{i, \text { out }}$, and therefore, her output pollutant concentration is always equal to this constant.

We denote the marginal cost of fresh water as $c[\$ / T]$, and the tax of discharged water as $\beta[\$ / T]$. Observe that, if the enterprise $i$ doesn't participate in the EIP, then her water consumption $z_{i}$ must be

$z_{i}=\frac{M_{i}}{C_{i, \text { out }}}$.

Then, the cost of her stand-alone operation, which we denote by $\mathrm{STC}_{i}[\$]$, is given by

$\mathrm{STC}_{i}=A \cdot(c+\beta) \frac{M_{i}}{C_{i, \text { out }}}$,

where $A[\mathrm{~h}]$ is a time constant that measures the lifetime of the park.

The goal of the authority is to built (and operate) an exchange network so part of this polluted water could be reused by other enterprises, minimizing the global consumption of fresh water within the park. Here, an exchange network for the EIP is a simple directed graph $\left(I_{0}, E\right)$, where the connection $(i, j) \in E$ means that the agent $i$ can send her output water to the agent $j$. In this sense, if agent $i$ uses the connection $(i, 0)$, then it means that she is discharging water outside the park, to the environment.

Defining the sets

$E_{\text {st }} \quad:=\left\{(i, 0): i \in I_{P}\right\}$

$E_{\max }:=\left\{(i, j): i \in I_{P}, j \in I_{0}\right\} \cup\left\{(r, j): r \in I_{R}, j \in I_{P}\right\}$,

a valid exchange network must satisfy that $E_{\text {st }} \subset E \subset E_{\text {max }}$. This definition yields that: (1) for every enterprise there is always the possibility of discharge; (2) the regeneration units can send water only to enterprises; and (3) the sink node doesn't have any exit connections (it is not possible to recover water once it is discharged). We denote by $\mathcal{E}$ the family of valid networks for the EIP. Finally, for any $E \in \mathcal{E}$, we denote by $E^{c}$ the set of connections that are not in $E$, that is, $E^{c}=E_{\max } \backslash E$.

Note that, on the one hand, the set $E_{\mathrm{st}}$ is the stand-alone configuration, where each enterprise only has access to fresh water and, after using it, she must discharge it to the sink node. On the other hand, $E_{\max }$ stands for the complete park, in the sense that all enterprises are connected between them, and all of them have access to the regeneration units.

If an enterprise $i \in I_{P}$ receives fluxes from other agents within the EIP, then these fluxed pass through a mixer. After the mixing, the inlet flux is then mixed with the purchased fresh water $z_{i}$. Then, the contaminant concentration of the total flux cannot surpass a limit inlet concentration $C_{i, \text { in }}$, which is given by technical constraints. We always have that $C_{i, \text { in }}<C_{i, \text { out }}$. This structure is illustrated in Fig. 5.1.

Every regeneration unit $r \in I_{R}$, as the enterprises, has limit concentrations as well. The inlet concentration for $r$ must be between a threshold given by a minimal inlet concentration, $C_{r, \text { in }}[\mathrm{ppm}]$. The output concentration $C_{r \text {,out }}$ [ppm] denotes the concentration of the output flux after the regeneration process. The main difference with the enterprises is that, while enterprises increase the contaminant concentration of fluxes, regeneration units reduce it. Therefore, we always have $C_{r \text {,out }} \leq C_{r \text {,in }}$. Fig. 5.2 illustrates the operation of regeneration units.

For each $(i, j) \in E_{\max }$ we denote by $F_{i, j}[\mathrm{~T} / \mathrm{h}]$ the water flux going from $i$ to $j$ through the connection $(i, j)$. We consider the following notation:

- $F_{i}=\left\{F_{i, j}: j \in I\right\}$ is the vector of fluxes exiting from agent $i$.

- $F_{-i}=\left(F_{k, j}: k \in I \backslash\{i\}\right)$ is the vector of all fluxes not exiting from agent $i$.

- $F_{-i}^{P}=\left(F_{k, j}: k \in I_{P} \backslash\{i\}\right)$ is the vector of all fluxes exiting from an enterprise different than $i$.

- $F^{R}=\left(F_{r}: r \in I_{R}\right)$ is the vector of fluxes exiting from regeneration units.

- $F^{P}=\left(F_{i}: i \in I_{P}\right)$ is the vector of fluxes exiting from enterprises.

Finally, for an agent $i \in I$, we may write $F=\left(F_{i}, F_{-i}\right)$, to stress the exiting fluxes of agent $i$. Moreover, if $i \in I_{P}$, we may also write

$F_{-i}=\left(F_{-i}^{P}, F^{R}\right) \quad$ and $\quad F=\left(F_{i}, F_{-i}^{P}, F^{R}\right)$,

to distinguish the actions of other enterprises and the actions of regeneration units. This is classic notation in game theory (see, e.g., Nisan et al., 2007; Ichiishi, 1983; Pang and Fukushima, 2005).

Then, for a fixed network $E$, a valid flux vector $F=\left(F_{i, j}\right.$ : $\left.(i, j) \in E_{\max }\right)$ must satisfy the following constraints:

1. Use of connections in $E$ : Since $E$ represents the available connections, we must put

$\forall(i, j) \in E^{c}, F_{i, j}=0$.

2. Water mass balance: Around an agent $i \in I$ (different from the sink node), we have that

$z_{i}+\sum_{(k, i) \in E} F_{k, i}=\sum_{(i, j) \in E} F_{i, j}$

If $i \in I_{R}$ then $z_{i}=0$, that is, the regeneration units don't consume fresh water. The sink node is not subject to balance constraints.

3. Contaminant mass balance: Around an enterprise $i \in I_{P}$, we have that

$M_{i}+\sum_{(k, i) \in E} C_{k, \text { out }} F_{k, i}=C_{i, \text { out }} \sum_{(i, j) \in E} F_{i, j}$,

where the right-hand term corresponds to the inlet contaminant mass coming from other agents, and the left-hand corresponds to the outlet contaminant mass. Thanks to the hypothesis of optimal response, the inlet mass of an agent can be expressed in terms of fluxes, since for each agent $k \in I$, the outlet concentration $C_{k, \text { out }}$ is always attained. Observe that regeneration units are not subject to contaminant mass balance, since they clean the water that pass through them.

4. Inlet/outlet concentration constraints: for an enterprise $i \in I_{P}$ we have that

$\sum_{(k, i) \in E} C_{k, \text { out }} F_{k, i} \leq C_{i, \text { in }}\left(z_{i}+\sum_{(k, i) \in E} F_{k, i}\right)$.

In parallel, for regeneration unit $r \in I_{R}$ we have that

$C_{r, \text { in }} \sum_{(k, r) \in E} F_{k, r} \leq \sum_{(k, r) \in E} C_{k, \text { out }} F_{k, r}$

Both constraints are formulated in terms of contaminant mass, but in practice they represent constraints of concentration.

5. Positivity of fluxes: all the fluxes in the park must be positive:

$$
\forall(i, j) \in E, F_{i, j} \geq 0 \quad \text { and } \quad \forall i \in I_{P}, z_{i} \geq 0 .
$$




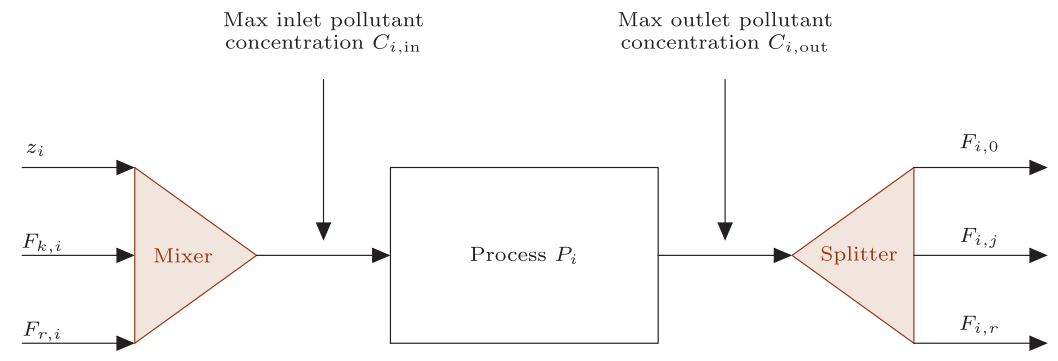

Fig. 5.1. Water mixture description for a given enterprise. Here $C_{i, \text { in }} \leq C_{i, \text { out }}$.

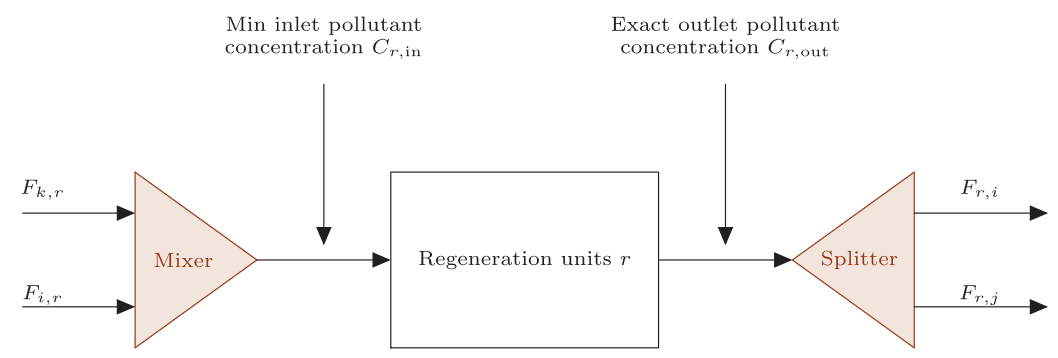

Fig. 5.2. Water mixture description for a given regeneration unit. Here $C_{r, \text { in }} \geq C_{t, \text { out }}$.The indexes $i, j, k$ belong to $I_{P}$.

Observe that, combining Eqs. (5.3) and (5.4) we obtain:

$M_{i}+\sum_{(k, i) \in E} C_{k, \text { out }} F_{k, i}=C_{i, \text { out }}\left(z_{i}+\sum_{(k, i) \in E} F_{k, i}\right), \quad \forall i \in I_{p}$,

and so, the fresh water bought by the enterprise $i \in I_{P}$ is given by the fluxes of the other agents, that is,

$z_{i}\left(F_{-i}\right)=\frac{1}{C_{i, \text { out }}}\left(M_{i}+\sum_{(k, i) \in E}\left(C_{k, \text { out }}-C_{i, \text { out }}\right) F_{k, i}\right)$.

In this model, each enterprise $i \in I_{P}$ has a cost function that she wants to minimize, defined by

$$
\begin{aligned}
& \operatorname{Cost}_{i}(F)=A\left[c \cdot z_{i}\left(F_{-i}\right)+\beta F_{i, 0}+2 \delta \sum_{r \in I_{R}}\left(F_{i, r}+F_{r, i}\right)\right. \\
& \left.+\delta \sum_{k, j \in I_{P}}\left(F_{k, i}+F_{i, j}\right)+\sum_{r \in I_{R}} \Gamma_{r} F_{r, i}^{\psi}\right],
\end{aligned}
$$

where $\Gamma_{r}[\$ / T]$ is the marginal cost of regenerating water depending on the technology of the regeneration unit and $\delta[\$ / T]$ is the marginal cost of using a sharing connection. Note that the regenerated water cost is non-linear, due to the power $\psi<1$, which we usually set between 0.6 and 0.8 (see e.g. Ramos et al., 2016). In fact the higher the volume of regenerated water, the lesser the operating cost of the regeneration unit. We assume that $\beta \gg 2 \delta$, that is, that the cost $\beta$ is much higher than $2 \delta$.

Observe also that each enterprise pays both for the entering water and the exiting water, for every connection she has. In the case of the connections between two enterprises, this means that the cost of the connection is divided uniformly between the sending enterprise and the receiving enterprise. In the case of regeneration units, the $2 \delta$ factor means that the enterprise must pay for the operation (when sending to and receiving from) of the regeneration unit.

On her part, the authority wants to minimize the consumption of the natural resources, and so she tries to minimize the function

$$
Z(F)=\sum_{i \in I_{P}} z_{i}\left(F_{-i}\right)
$$

All investment costs are trespassed to the enterprises by the marginal prices $\delta$ and $\Gamma_{r}$ and the exponent $\psi$.

In order to get each enterprise $i \in I_{P}$ to participate in the EIP, the authority engages in a Blind-Input contract with them of constant $\alpha \in] 0,1\left[\right.$. On the one hand, for each enterprise $i \in I_{P}$, the authority must ensure a relative improvement of $\alpha$ in the costs, with respect to the stand-alone operation, that is,

$\operatorname{Cost}_{i}\left(F_{i}, F_{-i}\right) \leq \alpha \mathrm{STC}_{i}$.

On the other hand, each enterprise commits to accept every inlet flux sent to her through the connections of the park, whenever these fluxes respect her physical constraints (which are given by (5.5)).

With all these considerations, for a given network $E \in \mathcal{E}$, the problem of each enterprise is given by problem $P_{i}\left(F_{-i}, E\right)$

$$
\begin{aligned}
& \min _{F_{i}} \operatorname{Cost}_{i}\left(F_{i}, F_{-i}\right) \\
& \text { s.t. }\left\{\begin{array}{l}
\text { Equations }(5.2)-(5.3)-(5.5), \\
z_{i}\left(F_{-i}\right) \geq 0, \\
F_{i} \geq 0 .
\end{array}\right.
\end{aligned}
$$

Observe that constraint (5.4) is implicit in the expression of $z_{i}\left(F_{-i}\right)$ given by (5.9). For a network $E \in \mathcal{E}$ and a fixed operation of the regeneration units $F^{R}$, we say that a vector $F^{P}$ is an equilibrium for the enterprises if and only if

$\forall i \in I_{P}, F_{i}$ solves the problem $P_{i}\left(F_{-i}, E\right)$.

We denote by $\operatorname{Eq}\left(F^{R}, E\right)$ the set of equilibria for $F^{R}$ and $E$. Then, the problem of the authority is

$$
\begin{aligned}
& \min _{F \in \mathbb{R}^{\left|E_{\max }\right|, E \in \mathcal{E}}} Z(F) \\
& \text { s.t. } \begin{cases}\sum_{(i, r) \in E} F_{i, r}=\sum_{(r, j) \in E} F_{r, j}, & \forall r \in I_{R}, \\
\sum_{(k, r) \in E} C_{k, \text { out }} F_{k, r} \leq C_{r, \text { in }}^{\max } \sum_{(k, r) \in E} F_{k, r}, & \forall r \in I_{R}, \\
C_{r, \text { in }}^{\min } \sum_{(k, r) \in E} F_{k, r} \leq \sum_{(k, r) \in E} C_{k, \text { out }} F_{k, r}, & \forall r \in I_{R}, \\
F_{r, j}=0, & \forall(r, j) \in E^{c}, r \in I_{R}, \\
F^{P} \in \operatorname{Eq}\left(F^{R}, E\right), & \\
F^{R} \geq 0, & \forall i \in I_{P} . \\
\operatorname{Cost}_{i}\left(F_{i}, F_{-i}\right) \leq \alpha \operatorname{STC}_{i}, & \end{cases}
\end{aligned}
$$


It is not hard to verify that Problem (5.14) together with the lower level problems (5.13) fit into the general Blind-Input model developed in Section 3. Indeed we only need to identify the variable $x$ with the flux vector $F$, to observe that the costs functions have the structure described in Section 3.3 and to note that constraint (5.5) can be rewritten with the form

$g_{i}\left(F_{-i}\right) \leq 0$

Thus, in the following, we will apply the reformulation described in Section 4.

\subsection{Problem formulation without regeneration units}

Now, we can reduce problem (5.14) to a Mixed-Integer programming problem. In this section, we analyze the case when there is no regeneration unit, that is, $I_{R}=\emptyset$.

To do so, we need to identify the pricing function $\gamma: E_{\max } \rightarrow$ $\mathbb{R}_{+}$which gives the marginal price of using exiting connections in the park and then, for each $i \in I_{P}$, we need to find the arc classes

$C(i, j)=\left\{(i, k) \in E_{\max }: \gamma(i, j)=\gamma(i, k)\right\}$.

Finally, we compute for each enterprise $i \in I_{P}$ the class set $\mathcal{C}_{i}=$ $\left\{C(i, j):(i, j) \in E_{\max }\right\}$.

Looking at the cost function (5.10) and assuming that there is no regeneration units, the pricing function $\gamma$ is given by

$\gamma(i, j)= \begin{cases}\delta & \text { if } j \in I_{P} \\ \beta & \text { if } j=0 .\end{cases}$

Thus, for each enterprise $i \in I_{P}$, the set $\mathcal{C}_{i}$ is equal to $\left\{C_{i, p}, C_{i, 0}\right\}$ where

$C_{i, p}=\left\{(i, j) \in E_{\max }: j \in I_{P}\right\}$

$C_{i, 0}=\{(i, 0)\}$.

Now, for each agent $i \in I_{P}$, we include three integer variables, $y_{i, p}, y_{i, 0}, y_{i, \text { null }} \in\{0,1\}$ with the following interpretation:

- If $y_{i, p}=1$, it means that the connections in $C_{i, p}$ are included in the network.

- If $y_{i, 0}=1$, it means that the connection $(i, 0)$ is the only exit connection for $i$, and $i$ participates in the EIP.

- If $y_{i, \text { null }}=1$, it means that the connection $(i, 0)$ is the only exit connection for $i$, and $i$ does not participate in the EIP (she works in stand-alone mode).

The main point is that only one of these variables takes the value 1 , and in doing so it determines the network $E$ to be implemented and the operation that each enterprises can do within this network. We denote by $y \in\{0,1\}^{3 n}$ the vector of all integer variables of all enterprises. If an enterprise $i$ is considered in the park, then Eq. (5.12) is active, but if not, then her cost coincides with $\mathrm{STC}_{i}$. Thus, we define the upper bound for the costs of enterprises as a function of the variable $y_{i, \text { null }}$ given by

$\operatorname{STC}_{i}\left(y_{i, \text { null }}\right)=\alpha \mathrm{STC}_{i} \cdot\left(1-y_{i, \text { null }}\right)+\mathrm{STC}_{i} \cdot y_{i, \text { null }}$.
Then, applying the reformulation of Section 4.3 we obtain the following problem:

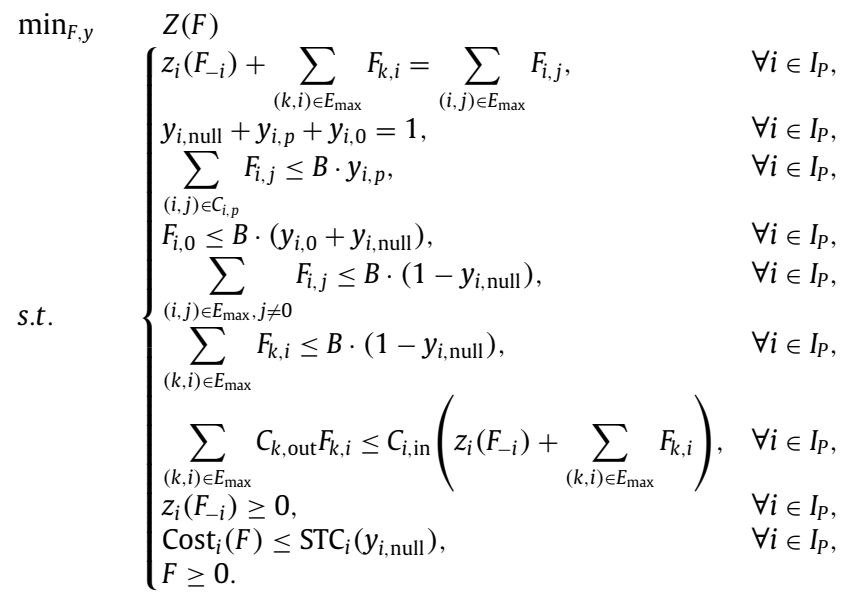

Here, $B$ is a constant large enough so all fluxes within the park, regardless the connections, are less than $B$. In practice, we set $B$ as

$B:=\sum_{i \in I_{P}} z_{i}(0)=\sum_{i \in I_{P}} \frac{M_{i}}{C_{i, \text { out }}}$,

that is, the total fresh water consumption assuming that each enterprise works in stand-alone operation.

\subsection{Problem formulation including regeneration units}

Let us now consider a network with regenaration units. Again, we need to identify the pricing function $\gamma: E_{\max } \rightarrow \mathbb{R}_{+}$and the class sets $\mathcal{C}_{i}$, for each enterprise $i \in I_{P}$. Recall that we don't need to work with classes for regeneration units, since they are controlled by the authority and so they don't have economic incentives.

The function $\gamma$ in this situation is given by

$\gamma(i, j)= \begin{cases}\delta & \text { if } j \in I_{P}, \\ 2 \delta & \text { if } j \in I_{R}, \\ \beta & \text { if } j=0 .\end{cases}$

Thus, for each enterprise $i \in I_{P}$, the set $\mathcal{C}_{i}=\left\{C_{i, p}, C_{i, r}, C_{i, 0}\right\}$ where

$C_{i, p}=\left\{(i, j) \in E_{\max }: j \in I_{P}\right\}$

$C_{i, r}=\left\{(i, r) \in E_{\max }: r \in I_{R}\right\}$

$C_{i, 0}=\{(i, 0)\}$.

As in Section 5.1, for each agent $i \in I_{P}$, we include four integer variables, $y_{i, p}, y_{i, r} y_{i, 0}, y_{i, \text { null }} \in\{0,1\}$ with the following interpretation:

- If $y_{i, p}=1$, it means that the connections in $C_{i, p}$ are included in the network.

- If $y_{i, r}=1$, it means that the connections in $C_{i, r}$ are included in the network.

- If $y_{i, 0}=1$, it means that the connection $(i, 0)$ is the only exit connection for $i$, and $i$ participates in the EIP.

- If $y_{i, \text { null }}=1$, it means that the connection $(i, 0)$ is the only exit connection for $i$, and $i$ does not participate in the EIP (she works in stand-alone mode).

Again, only one of this variables can take the value 1 . Keeping the same notation $y \in\{0,1\}^{4 n}$ for the complete vector of integer variables, setting $\operatorname{STC}_{i}\left(y_{i, \text { null }}\right)$ and $B$ as in definitions (5.16) and (5.18), respectively, and applying the reformulation of Section 4.3, 
Table 2

Enterprises' parameters.

\begin{tabular}{llll}
\hline Enterprise $i$ & $\mathrm{C}_{i, \text { in }}(\mathrm{ppm})$ & $\mathrm{C}_{i, \text { out }}(\mathrm{ppm})$ & $\mathrm{M}_{i}(\mathrm{~g} / \mathrm{h})$ \\
\hline 1 & 0 & 100 & 7500 \\
2 & 0 & 200 & 6000 \\
3 & 50 & 100 & 5000 \\
4 & 80 & 800 & 30,000 \\
5 & 400 & 800 & 4000 \\
6 & 20 & 100 & 2500 \\
7 & 50 & 100 & 2200 \\
8 & 80 & 400 & 5000 \\
9 & 100 & 800 & 30,000 \\
10 & 400 & 1000 & 4000 \\
11 & 30 & 60 & 2000 \\
12 & 25 & 50 & 2000 \\
13 & 25 & 75 & 5000 \\
14 & 50 & 800 & 30,000 \\
15 & 100 & 200 & 13,000 \\
\hline
\end{tabular}

we obtain the following problem:

$\begin{array}{ll}\min _{F, y}(y) & \forall i \in I_{P}, \\ z_{i}\left(F_{-i}\right)+\sum_{(k, i) \in E_{\max }} F_{k, i}=\sum_{(i, j) \in E_{\max }} F_{i, j}, & \forall r \in I_{R}, \\ \sum_{(k, r) \in E_{\max }} F_{k, r}=\sum_{(r, j) \in E_{\max }} F_{r, j}, & \forall i \in I_{P}, \\ y_{i, \text { null }}+y_{i, p}+y_{i, r}+y_{i, 0}=1, & \forall i \in I_{P}, \\ \sum_{(i, j) \in C_{i, p}} F_{i, j} \leq B \cdot y_{i, p}, & \forall i \in I_{P}, \\ \sum_{(i, j) \in C_{i, r}} F_{i, j} \leq B \cdot y_{i, r}, & \forall i \in I_{P}, \\ F_{i, 0} \leq B \cdot\left(y_{i, 0}+y_{i, \text { null }}\right), & \forall i \in I_{P}, \\ \sum_{(i, j) \in E_{\max }, j \neq 0} F_{i, j} \leq B \cdot\left(1-y_{i, \text { null }}\right), & \forall i \in I_{P}, \\ \sum_{(k, i) \in E_{\max }} F_{k, i} \leq B \cdot\left(1-y_{i, \text { null }}\right), & \forall i \in I_{P}, \\ \sum_{(k, i) \in E_{\max }} C_{k, \text { out }} F_{k, i} \leq C_{i, \text { in }}\left(z_{i}\left(F_{-i}\right)+\sum_{(k, i) \in E_{\max }} F_{k, i}\right) & \forall r \in I_{R}, \\ \sum_{(r, k) \in E_{\max }} C_{r, \text { in }}^{\min } F_{r, k}-\sum_{(k, r) \in E_{\max }} C_{k, \text { out }} F_{k, r} \leq 0, & \forall r \in I_{R}, \\ \sum_{(k, r) \in E_{\max }} C_{k, \text { out }} F_{k, r}-\sum_{(r, k) \in E_{\max }}^{\max } C_{r, \text { in }} F_{r, k} \leq 0, & \forall i \in I_{P}, \\ z_{i}\left(F_{-i} \geq 0,\right. & \forall i \in I_{P}, \\ \operatorname{Cost}_{i}(F) \leq \alpha \cdot \operatorname{STC}_{i}\left(y_{i, \text { null }}\right), & \\ F \geq 0 . & \end{array}$

\section{Simulation with some academic examples}

In this section we present numerical examples of the BlindInput model applied to water exchange networks in Eco-Industrial Parks. The detailed model we use is described in Section 5. The optimization problems we solve correspond to adaptations of problem (4.10) to the EIP: specifically, we solve problem (5.17) for parks without regeneration units, and problem (5.20) for parks with regeneration units.

The study case we present consists on an EIP made up of 15 enterprises, each one including only one process, and 3 regeneration units. Data is partially inspired from Olesen and Polley (1996); Ramos et al. (2016). It is assumed that the EIP operates for one hour, that is, $A=1 \mathrm{~h}$. We consider three different regeneration units which differ by their capacity to regenerate water. Concerning the power $\psi$, we fix $\psi=0.6$.

The data of 15 enterprises is given in Table 2. Prices are shown in Table 3. In addition, the operating parameters of regeneration units are illustrated in Table 4 . In terms of concentration limits, we set $C_{r \text {,out }}=C_{r \text {,in }}$ for each $r \in I_{R}$, to simplify the problem.
Table 3

Associated costs.

\begin{tabular}{ll}
\hline Parameter & Value (\$/tonne) \\
\hline$c$ & 0.13 \\
$\beta$ & 0.22 \\
$\delta$ & 0.01 \\
\hline
\end{tabular}

Table 4

Different regeneration units. We suppose $C_{r \text {, in }}=$ $C_{r, \text { out }}$.

\begin{tabular}{lll}
\hline Unit $r$ & $C_{r, \text { out }}=C_{r, \text { in }}(\mathrm{ppm})$ & $\Gamma_{r}(\$ /$ tonne $)$ \\
\hline 1 & 30 & 0.85 \\
2 & 40 & 0.695 \\
3 & 50 & 0.54 \\
\hline
\end{tabular}

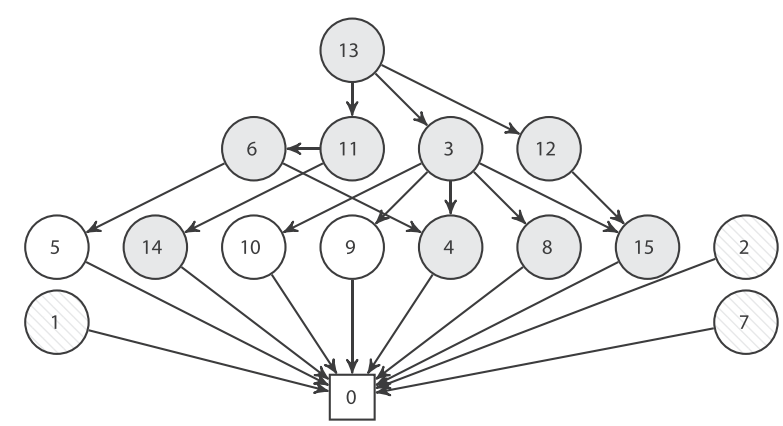

Fig. 6.1. The configuration in the case without regeneration units, $\alpha=0.95$ and Coef $=1$. Gray nodes are consuming strictly positive fresh water. Dashed nodes are operating in stand-alone mode.

Since the optimization problems we solve (problems (5.17) and (5.20)) may have several solutions (see Section 6.3 for an example), we introduce a penalization term in the objective function in order to obtain the one having more participating enterprises. We replace $Z(F)$ by

$Z(F)+$ Coef $\cdot \sum_{i \in I_{P}} y_{i, \text { null }}$,

where Coef $\geq 0$ is a coefficient to penalize those optimal solutions that leave more enterprises outside the park. If Coef is too large, we may sacrifice optimality of fresh water consumption by forcing enterprises into the park. Numerical experiments show that Coef $=1$ is a good value. The selection and impact of this coefficient should be further studied but such analysis is out the scope of the article.

All simulations where implemented with Julia v1.2.0 programming language (Bezanson et al., 2017), using Gurobi v8.1.1 as solver (LLC Gurobi Optimization, 2020).

\subsection{Numerical experiment without regeneration units}

Here, we present the results of the simulations with the data exposed before. The optimized configuration of the EIP is presented in Fig. 6.1, and it corresponds to a Blind-Input contract with $\alpha=0.95$ and Coef $=1$. Detailed results are summarized in Table 5. Detailed results of fluxes within the network are presented in Table A.10.

Of course, the optimization results are sensible to the parameter $\alpha$ chosen for the Blind-Input contract. Fig. 6.2 shows the results when considering $\alpha \in[0.5,0.99]$, in terms of total fresh water consumption and the number of enterprises left out of the EIP, that is, the number of stand-alone enterprises. For values of $\alpha$ smaller than 0.5 , it would mean that the authority is offering an improve- 
Table 5

Summary of results of the EIP without regeneration units. Marked enterprises $\left(^{*}\right)$ are left outside the park, operating stand-alone.

\begin{tabular}{lllll}
\hline Enterprise & Fresh water stand-alone & Fresh water in EIP & Cost $_{i}$ stand-alone & Cost $_{i}$ in EIP \\
\hline $1^{*}$ & 75.00 & 75.00 & 26.25 & 26.25 \\
$2^{*}$ & 30.00 & 30.00 & 10.50 & 10.50 \\
3 & 50.00 & 42.08 & 17.50 & 6.52 \\
4 & 37.50 & 8.33 & 13.13 & 10.58 \\
5 & 5.00 & 0.00 & 1.75 & 1.31 \\
6 & 25.00 & 20.83 & 8.75 & 3.13 \\
$7^{*}$ & 22.00 & 22.00 & 7.70 & 7.70 \\
8 & 12.50 & 3.13 & 4.38 & 3.97 \\
9 & 37.50 & 0.00 & 13.13 & 9.86 \\
10 & 4.00 & 0.00 & 1.40 & 1.02 \\
11 & 33.33 & 35.42 & 11.67 & 5.13 \\
12 & 40.00 & 53.33 & 14.00 & 8.00 \\
13 & 66.67 & 66.67 & 23.33 & 9.33 \\
14 & 37.50 & 6.66 & 13.13 & 10.00 \\
15 & 65.00 & 1.92 & 22.75 & 20.49 \\
Total & 541.00 & 365.37 & 189.37 & 133.79 \\
\hline
\end{tabular}

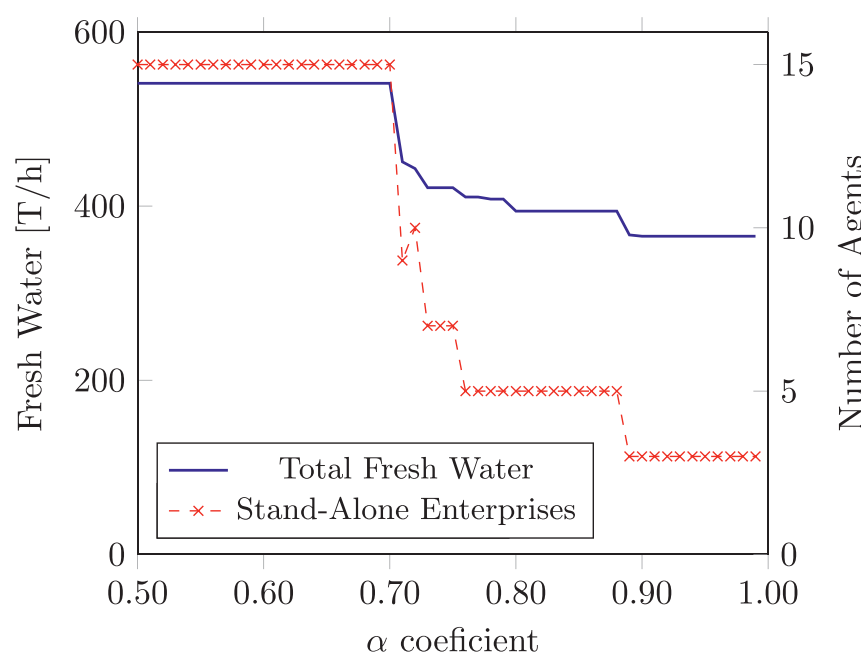

Fig. 6.2. Sensitivity Analysis for $\alpha \in[0.50,0.99]$ and Coef $=1$. Total fresh water consumption and number of stand-alone enterprises.

ment of more than $50 \%$ to each participant of the park, which is unrealistic.

\subsection{Numerical experiment including regeneration units}

The main difficulty to solve Problem (5.20) is that the function Cost $_{i}$ described in (5.10) is nonlinear under the presence of Regeneration Units. To tackle this obstruction, for each unit $r \in I_{R}$ we construct a piecewise linear approximation of the functions $f_{r}: x_{\mapsto} \Gamma_{r} \cdot x^{\psi}$, which is the marginal costs of regenerating water. This approximation, that we denote $\ell_{r}$, is constructed in the interval $[0, B]$ (where $B$ is the constant given in (5.18)), with the reference points $S=\left(s_{0}, s_{1}, s_{2}, s_{3}, s_{4}, s_{5}\right)=(0,0.1 B, 0.2 B, 0.4 B, 0.6 B, B)$. Fig. 6.3 shows the approximations $\ell_{r}$ for the regeneration units described in Table 4.

The nonuniform partition of the interval $[0, B]$ is due to the fact that $B$ is not a tight bound: each value $F_{r, j}$ with $r \in I_{R}$ and $i \in I_{P}$ should be a lot less than $B$. Thus, the approximation $\ell_{r}$ must be more precise in the first part of the interval.

As we did in Section 6.1, we present the detailed results of the simulations for $\alpha=0.95$ and Coef $=1$ in Table 6 . The obtained configuration of the park is given in Fig. 6.1. Detailed results of fluxes within the network are presented in Table A.11.

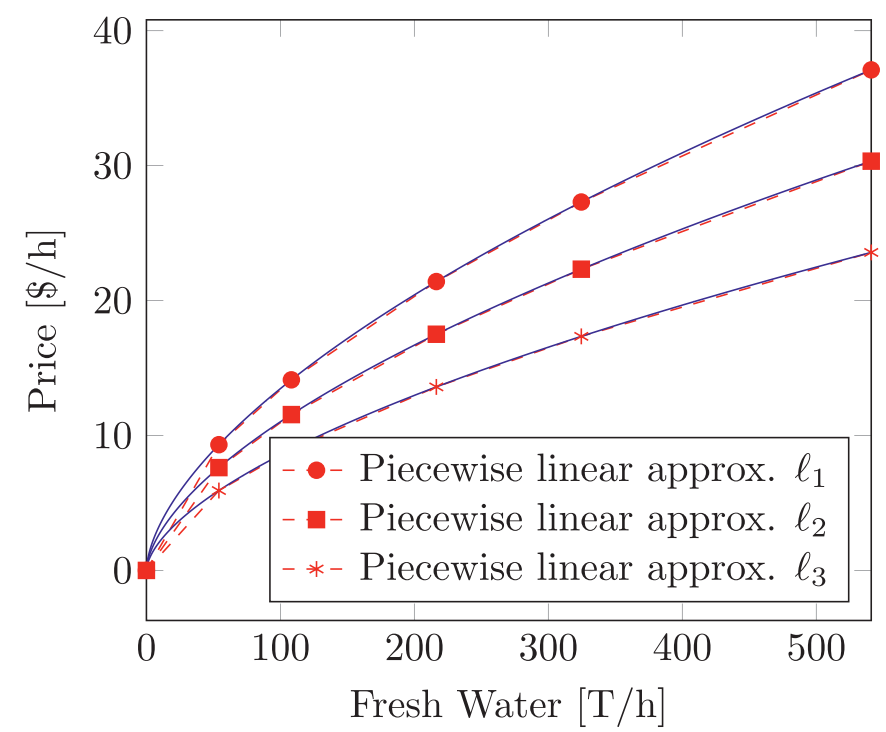

Fig. 6.3. Piecewise linear approximations (dashed red) of marginal cost of regenerating water. The function $\ell_{r}$ corresponds to Reg. Unit $r \in I_{R}=\{1,2,3\}$. The original function $f_{r}$ is given in blue. The parameters of $f_{r}$ correspond to the data presented at the beginning of Section 6. . (For interpretation of the references to color in this figure legend, the reader is referred to the web version of this article.)

Finally, the sensitivity analysis of the results with respect to $\alpha \in[0.5,0.99]$ is shown in Fig. 6.5, again, showing the variation of total fresh water consumption and the number of stand-alone enterprises. As in the case without regeneration units, we neglect values of $\alpha$ smaller than 0.5 due to the impossibility of implement such a contract (Fig. 6.4).

\subsection{Small case with multiple solutions}

In this last part, we present an small example to show that problem (5.17) may have multiple optimal solutions. This example presents two optimal solutions that have different number of participating enterprises. The case of study consists on an EIP made up of 5 enterprises described in Table 7 . The prices of the example are the same as before, presented in Table 3, and the Blind-Input parameter is fixed as $\alpha=0.99$.

Given that the example is small, we were able to explore all combinations of participating enterprises: each time, we chose a subset $A \subset I_{P}=\{1,2,3,4,5\}$ and we solved problem (5.17) with the 
Table 6

Summary of results of the EIP considering regeneration units with Coef $=1$.

\begin{tabular}{lllll}
\hline Enterprise & Fresh water stand-alone & Fresh water in EIP & Cost $_{i}$ stand-alone & Cost $_{i}$ in EIP \\
\hline 1 & 75.00 & 75.00 & 26.25 & 10.50 \\
2 & 30.00 & 30.00 & 10.50 & 4.20 \\
3 & 50.00 & 0.00 & 17.50 & 11.16 \\
4 & 37.50 & 0.00 & 13.13 & 5.58 \\
5 & 5.00 & 0.00 & 1.75 & 1.53 \\
6 & 25.00 & 10.42 & 8.75 & 5.98 \\
7 & 22.00 & 0.00 & 7.70 & 6.50 \\
8 & 12.50 & 0.00 & 4.38 & 12.47 \\
10 & 4.00 & 0.00 & 1.40 & 0.15 \\
11 & 33.33 & 7.15 & 11.67 & 11.08 \\
12 & 40.00 & 18.94 & 14.00 & 13.30 \\
13 & 66.67 & 16.67 & 23.33 & 17.75 \\
14 & 37.50 & 0.00 & 13.13 & 6.41 \\
15 & 65.00 & 0.00 & 22.75 & 21.40 \\
Total & 541.00 & 158.17 & 189.35 & 132.17 \\
\hline
\end{tabular}

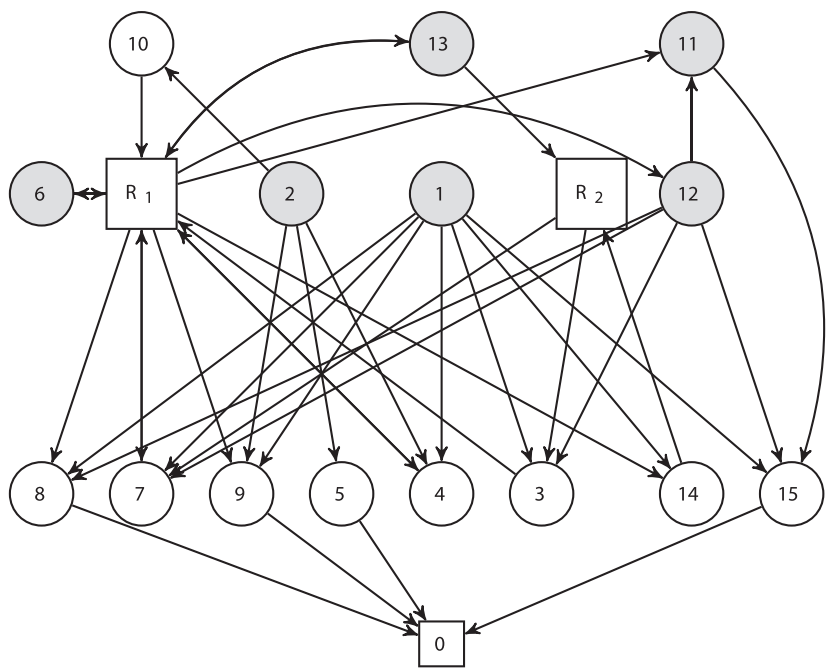

Fig. 6.4. The configuration considering regeneration units, $\alpha=0.95$ and Coef $=1$. Gray nodes are consuming strictly positive fresh water. Regeneration unit $R_{3}$ is not used and therefore removed from the park.

Table 7

Enterprises' parameters for small size case of study.

\begin{tabular}{llll}
\hline Enterprise & $\mathrm{C}_{i, \text { in }}(\mathrm{ppm})$ & $\mathrm{C}_{i, \text { out }}(\mathrm{ppm})$ & $\mathrm{M}_{p}(\mathrm{~g} / \mathrm{h})$ \\
\hline 1 & 175 & 898 & 30,010 \\
2 & 90 & 200 & 3000 \\
3 & 30 & 35 & 1000 \\
4 & 150 & 530 & 32,030 \\
5 & 400 & 1095 & 90,000 \\
\hline
\end{tabular}

extra constraint

$y_{i, \text { null }}= \begin{cases}0 & \text { if } i \in A \\ 1 & \text { otherwise }\end{cases}$

This exhaustive exploration can be done only because the set of enterprises is small, since the number of possible subsets $A \subset I_{P}$ is $2^{\left|I_{P}\right|}$.

After exploring all possible combination, we found two different optimal configurations that are shown in Figs. 6.6 and 6.7.

The results of fresh water consumption and economic cost are summarized in Tables 8 and 9.

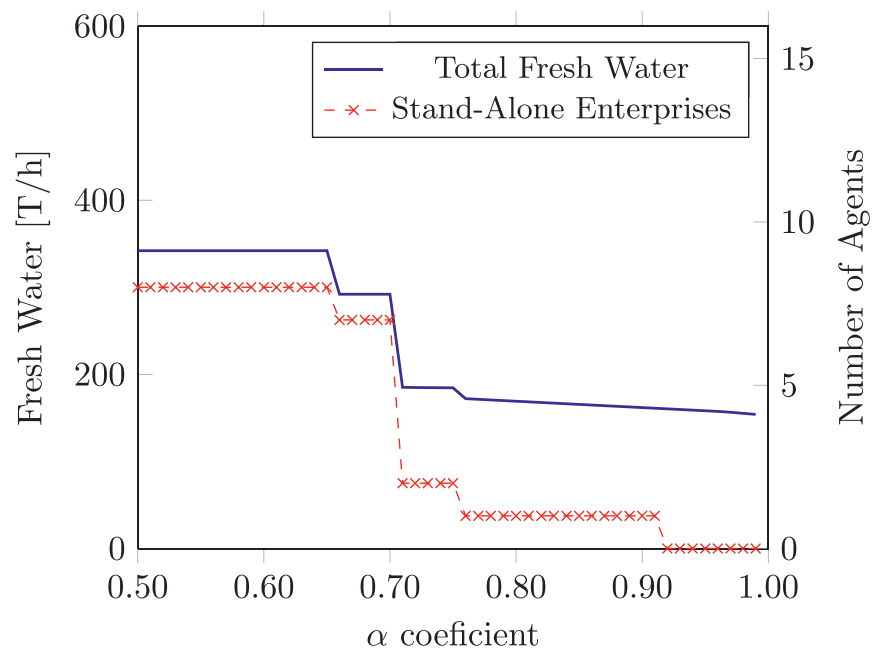

Fig. 6.5. Sensitivity Analysis for $\alpha \in[0.50,0.99]$ and Coef $=1$. Total fresh water consumption and number of stand-alone enterprises.

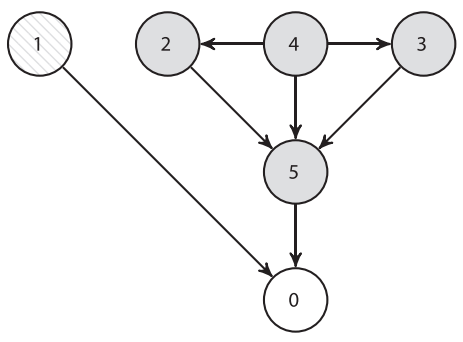

Fig. 6.6. The configuration in the case without regeneration units, $\alpha=0.99$. Gray nodes are consuming strictly positive fresh water.

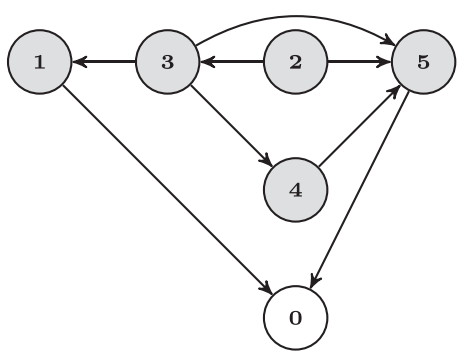

Fig. 6.7. The configuration in the case without regeneration units, $\alpha=0.99$. Gray nodes are consuming strictly positive fresh water. 
Table 8

Fresh water consumption $[\mathrm{T} / \mathrm{h}]$. Stand-alone configuration and optimal solutions. In the first solution, Enterprise 1 is left in standalone operation.

\begin{tabular}{llll}
\hline Enterprise & Stand-alone & First solution & Second solution \\
\hline 1 & 33.42 & $33.42^{*}$ & 32.36 \\
2 & 15 & 18.65 & 15 \\
3 & 28.57 & 33.98 & 64.68 \\
4 & 60.43 & 60.43 & 62.26 \\
5 & 82.19 & 2.03 & 0 \\
Total & 219.61 & 148.51 & 148.51 \\
\hline
\end{tabular}

\section{Table 9}

Economic cost $[\$ / \mathrm{h}]$. Stand-alone configuration and optimal solutions. In the first solution, Enterprise 1 is left in stand-alone operation.

\begin{tabular}{llll}
\hline Enterprise & Stand-alone & First solution & Second solution \\
\hline 1 & 11.70 & $11.70^{*}$ & 11.58 \\
2 & 5.25 & 2.65 & 2.1 \\
3 & 10 & 4.76 & 8.30 \\
4 & 21.15 & 8.46 & 5.39 \\
5 & 28.77 & 26.71 & 27.45 \\
Total & 76.87 & 54.28 & 54.82 \\
\hline
\end{tabular}

\section{Discussion}

The results presented for our academic example, in both cases without and with regeneration units, show that optimal networks under Blind-Input contracts provide substantial improvements in terms of reducing the global consumption of water and the cost of each participating enterprise. Relatively speaking, the EIP without regeneration units reduces the amount of consumed water in $32 \%$ with respect to the stand-alone configuration, while the EIP with regeneration units reduces it is more than $70 \%$.

Moreover, each participating enterprise has a cost reduction of at least $5 \%$, as promised by the Blind-Input contract. Exploring both, Tables 5 and 6 , the reader can observe that the reduction is not uniform among the enterprises. This unbalanced benefit is due to the nature of the problem: the authority is only concerned by the total water consumption, and therefore, the costs of the enterprises is not relevant beyond the Blind-Input contract. This is acceptable recalling that the Blind-Input model maintains the information of each enterprise private.

From the perspective of each enterprise, she only interacts economically with the authority and so she only perceives her gains. It is therefore economically appealing to participate in the EIP under the network proposed by the authority. However, the model presented in this work may not be economically efficient in the sense that the total cost for the enterprises may not be optimal. Fig. 7.1 shows a sensitivity analysis of total cost for enterprises depending on the Blind-Input parameter $\alpha \in[0.50,0.99]$.

One would expect that total cost, at least starting from some threshold where enough enterprises participate into the park, should be increasing with respect to $\alpha$, since larger the $\alpha$, less exigent are the enterprises. However, this is not the case. The behavior of the total cost is quite chaotic, which is more evident for the case with regeneration units. In terms of total cost of enterprises, the only behavior that is natural is that the influence of regeneration units not only reduces the total water consumption, but also the total cost the enterprises.

This behavior in economic cost can be explained by the multiplicity of optimal configurations. The small size example presented in Section 6.3 emphasizes that there may be several optimal configurations in terms of total water consumption, differing in the number of stand-alone agents and total cost of enterprises. An interesting question rising from this example and the behavior of

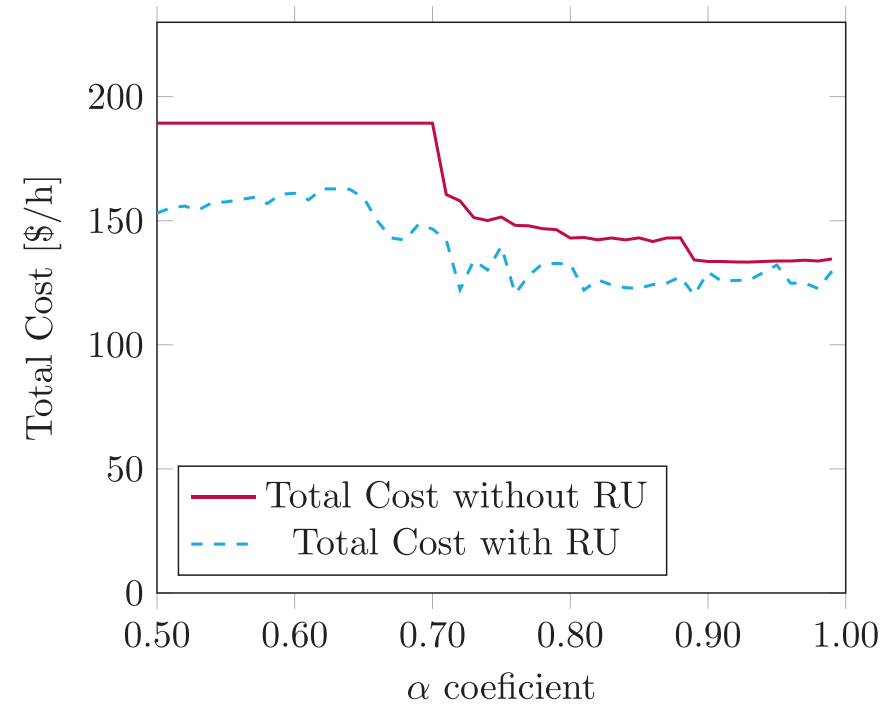

Fig. 7.1. Sensitivity Analysis for $\alpha \in[0.50,0.99]$ and Coef $=1$. Total cost of the park for both study cases: without and with regeneration units.

costs showed in Fig. 7.1 is how to select the "best" optimal configuration.

In order to minimize the number of stand-alone enterprises we introduce the penalization (6.1). However, some other penalization could be considered to try to force economic efficiency. In real-size problems (more than 10 enterprises), it is not possible to explore all configurations, and therefore penalized objective functions seem to be the solution. This could also be regarded in the sense of multi-objective optimization: the authority could consider not only the total water consumption, but also the number of stand-alone enterprises and the economic efficiency as optimization criteria. However, this could be delicate, since it is important to keep the minimization of fresh water consumption as the primary goal of the park. In the case of study without regeneration units, Fig. 6.2 shows that the minimization of stand-alone enterprises could be relegated for a better solution in terms of water consumption: for $\alpha=0.71,9$ enterprises are left in stand-alone operation, while for $\alpha=0.72,10$ enterprises are left in stand-alone operation. While this could be interpreted as an ill behavior of the algorithm, it is completely correct. Since $\alpha=0.72$ is bigger, the authority has more freedom to built the optimal network, and while she could implement the same solution that for $\alpha=0.71$, she chooses to left one extra enterprise outside of the park, and then reducing the fresh water consumption from $450.79[\mathrm{~T} / \mathrm{h}]$ (the optimal value for $\alpha=0.71$ ) to 443.08 [T/h] (the optimal value for $\alpha=0.72$ ).

This same example shows another limitation for the Blind-Input contract: on the one hand, if enterprises are too exigent ( $\alpha$ small), the authority may be incapable to find a economically feasible configuration. In fact, for $\alpha \leq 0.70$, the optimal solution is the standalone configuration, which means that the authority cannot build a park that attracts the exigent agents and also that reduces the total fresh water consumption. On the other hand, if enterprises are too polluting, they may not be able to participate in the park, even without economic constraints. Indeed, starting from $\alpha=0.89$, the authority leaves three enterprises outside the park, since their participation doesn't contribute to the minimization of fresh water. This is solved only by introducing regeneration units, where full participation is attained starting from $\alpha=0.92$. 


\section{Conclusion and perspectives}

Models for general exchange could lead to quite difficult problems like single-leader-multi-follower problems. In this work, our aim is to emphasize that under the Blind-Input paradigm which is economically consistent, one can find some solutions by simply solving a mixed-integer linear problem. This clearly allows to tackle large scale problems efficiently and propose exchange politics that attract enterprises to participate.

In order to apply the Blind-Input model to an exchange network, the following main elements must be present: (1) for each independent agent of the park, Kirchoff's law and positivity of fluxes must be the only constraints of the her optimization problem that include their own variables (i.e., the vector $x_{i}$ ); (2) the quality of each inlet flux must depend only on the sending agent; and (3) the optimal response hypothesis must allow each agent to compute the amount of natural resources needed. These three conditions are present in many other examples. Particularly, energy networks also fit this profile (see, e.g., Boix et al., 2015; Neves et al., 2020). Under these conditions, our general model of Section 3 (and of course the reductions and methods of Section 4) can be adapted and so, the network can be designed/operated following the Blind-Input paradigm. It is important to note that the economic model is a design decision, suitable for Eco-Industrial Parks involving noncooperative enterprises.

Of course, the Blind-Input paradigm can appear to be quite restrictive since followers are forced to accept the incoming fluxes. While this aspect is compensated by the Blind-Input contract, it could be interesting for a future work to consider exchange models in which agents could have more "control" on their exchanges. Taking advantage of the MILP formulation, some other theoretical results could be deduced on the exchange models; for example, stability results, dual formulations, among others.

In terms of the physical model, several other developments can be considered for future research. In this work, we only treat enterprises with single processes. Multi-process agents should also be explored. Specifically in terms of the water exchange model, we only consider a single-pollutant model. Multi-pollutant exchange networks (where enterprises must dilute different types of pollutants) introduce a particular difficulty with the inlet and outlet concentrations. The optimal response hypothesis changes, since each enterprise will have several outlet concentrations (one for each pollutant) to observe, entailing that these concentrations would become variable, changing the contaminant mass balance constraints as well as the formula for the fresh water consumption. A concrete perspective of this work is to include the multi-contaminant problem coupled with the energy network, following the developments of Almaraz et al. (2016). Finally, EcoIndustrial Parks with several exchange networks could be explored as well under the Blind-Input paradigm, following the spirit of Ramos et al. (2018b).

\section{Declaration of Competing Interest}

The authors declare that they have no known competing financial interests or personal relationships that could have appeared to influence the work reported in this paper.

\section{CRediT authorship contribution statement}

David Salas: Conceptualization, Writing - original draft, Writing - review \& editing. Kien Cao Van: Investigation, Writing - review \& editing. Didier Aussel: Supervision, Writing - review \& editing. Ludovic Montastruc: Supervision.

\section{Acknowledgments}

This work was done during a shared post-doctorate of the first author at Laboratoire de Génie Chimique of Toulouse, and Laboratoire PROMES of Perpignan, funded under the grant "Projet ANR JCJC-16-CE10-0001-01 - GREENSCOPE". The second author is sponsored by the "Région Occitanie" and Europe (through the Fonds européen de développement régional (FEDER)) while the research of the third author is partially granted by the "Fondation Mathématique Jacques Hadamard" throught the PGMO project.

\section{Appendix A. Network fluxes of simulations}

The values of the flux corresponding to the case without and with regeneration unit in Sections 6.1 and 6.2 are given in Tables A.10 and A.11, respectively. The entrance $(i, j)$ of both tables corresponds to the flux sent from agent $i$ to agent $j$.

Table A1

The values of the flux in the case without regeneration units, $\alpha=0.95$ and Coef $=1$.

\begin{tabular}{|c|c|c|c|c|c|c|c|c|c|c|c|c|c|c|c|c|}
\hline Enterprise & 1 & 2 & 3 & 4 & 5 & 6 & 7 & 8 & 9 & 10 & 11 & 12 & 13 & 14 & 15 & Sink \\
\hline 1 & 0.00 & 0.00 & 0.00 & 0.00 & 0.00 & 0.00 & 0.00 & 0.00 & 0.00 & 0.00 & 0.00 & 0.00 & 0.00 & 0.00 & 0.00 & 75.00 \\
\hline 2 & 0.00 & 0.00 & 0.00 & 0.00 & 0.00 & 0.00 & 0.00 & 0.00 & 0.00 & 0.00 & 0.00 & 0.00 & 0.00 & 0.00 & 0.00 & 30.00 \\
\hline 3 & 0.00 & 0.00 & 0.00 & 7.79 & 0.00 & 0.00 & 0.00 & 12.50 & 42.86 & 4.44 & 0.00 & 0.00 & 0.00 & 0.00 & 6.15 & 0.00 \\
\hline 4 & 0.00 & 0.00 & 0.00 & 0.00 & 0.00 & 0.00 & 0.00 & 0.00 & 0.00 & 0.00 & 0.00 & 0.00 & 0.00 & 0.00 & 0.00 & 41.67 \\
\hline 5 & 0.00 & 0.00 & 0.00 & 0.00 & 0.00 & 0.00 & 0.00 & 0.00 & 0.00 & 0.00 & 0.00 & 0.00 & 0.00 & 0.00 & 0.00 & 5.71 \\
\hline 6 & 0.00 & 0.00 & 0.00 & 25.54 & 5.71 & 0.00 & 0.00 & 0.00 & 0.00 & 0.00 & 0.00 & 0.00 & 0.00 & 0.00 & 0.00 & 0.00 \\
\hline 7 & 0.00 & 0.00 & 0.00 & 0.00 & 0.00 & 0.00 & 0.00 & 0.00 & 0.00 & 0.00 & 0.00 & 0.00 & 0.00 & 0.00 & 0.00 & 22.00 \\
\hline 8 & 0.00 & 0.00 & 0.00 & 0.00 & 0.00 & 0.00 & 0.00 & 0.00 & 0.00 & 0.00 & 0.00 & 0.00 & 0.00 & 0.00 & 0.00 & 15.63 \\
\hline 9 & 0.00 & 0.00 & 0.00 & 0.00 & 0.00 & 0.00 & 0.00 & 0.00 & 0.00 & 0.00 & 0.00 & 0.00 & 0.00 & 0.00 & 0.00 & 42.86 \\
\hline 10 & 0.00 & 0.00 & 0.00 & 0.00 & 0.00 & 0.00 & 0.00 & 0.00 & 0.00 & 0.00 & 0.00 & 0.00 & 0.00 & 0.00 & 0.00 & 4.44 \\
\hline 11 & 0.00 & 0.00 & 0.00 & 0.00 & 0.00 & 10.42 & 0.00 & 0.00 & 0.00 & 0.00 & 0.00 & 0.00 & 0.00 & 33.33 & 0.00 & 0.00 \\
\hline 12 & 0.00 & 0.00 & 0.00 & 0.00 & 0.00 & 0.00 & 0.00 & 0.00 & 0.00 & 0.00 & 0.00 & 0.00 & 0.00 & 0.00 & 80.00 & 0.00 \\
\hline 13 & 0.00 & 0.00 & 31.67 & 0.00 & 0.00 & 0.00 & 0.00 & 0.00 & 0.00 & 0.00 & 8.33 & 26.67 & 0.00 & 0.00 & 0.00 & 0.00 \\
\hline 14 & 0.00 & 0.00 & 0.00 & 0.00 & 0.00 & 0.00 & 0.00 & 0.00 & 0.00 & 0.00 & 0.00 & 0.00 & 0.00 & 0.00 & 0.00 & 40.00 \\
\hline 15 & 0.00 & 0.00 & 0.00 & 0.00 & 0.00 & 0.00 & 0.00 & 0.00 & 0.00 & 0.00 & 0.00 & 0.00 & 0.00 & 0.00 & 0.00 & 88.08 \\
\hline
\end{tabular}


Table A2

The values of the flux in the case with regeneration units, $\alpha=0.95$ and Coef $=1$.

\begin{tabular}{|c|c|c|c|c|c|c|c|c|c|c|c|c|c|c|c|c|c|c|c|}
\hline $\begin{array}{l}\text { Enterprise } \\
\text { and regen- } \\
\text { eration } \\
\text { units }\end{array}$ & 1 & 2 & 3 & 4 & 5 & 6 & 7 & 8 & 9 & 10 & 11 & 12 & 13 & 14 & 15 & $R_{1}$ & $R_{2}$ & $R_{3}$ & Sink \\
\hline 1 & 0.00 & 0.00 & 10.82 & 9.60 & 0.00 & 0.00 & 8.31 & 10.61 & 18.50 & 0.00 & 0.00 & 0.00 & 0.00 & 11.43 & 5.74 & 0.00 & 0.00 & 0.00 & 0.00 \\
\hline 2 & 0.00 & 0.00 & 0.00 & 8.30 & 6.67 & 0.00 & 0.00 & 0.00 & 10.03 & 5.00 & 0.00 & 0.00 & 0.00 & 0.00 & 0.00 & 0.00 & 0.00 & 0.00 & 0.00 \\
\hline 3 & 0.00 & 0.00 & 0.00 & 0.00 & 0.00 & 0.00 & 0.00 & 0.00 & 0.00 & 0.00 & 0.00 & 0.00 & 0.00 & 0.00 & 0.00 & 100.00 & 0.00 & 0.00 & 0.00 \\
\hline 4 & 0.00 & 0.00 & 0.00 & 0.00 & 0.00 & 0.00 & 0.00 & 0.00 & 0.00 & 0.00 & 0.00 & 0.00 & 0.00 & 0.00 & 0.00 & 41.67 & 0.00 & 0.00 & 0.00 \\
\hline 5 & 0.00 & 0.00 & 0.00 & 0.00 & 0.00 & 0.00 & 0.00 & 0.00 & 0.00 & 0.00 & 0.00 & 0.00 & 0.00 & 0.00 & 0.00 & 0.00 & 0.00 & 0.00 & 6.67 \\
\hline 6 & 0.00 & 0.00 & 0.00 & 0.00 & 0.00 & 0.00 & 0.00 & 0.00 & 0.00 & 0.00 & 0.00 & 0.00 & 0.00 & 0.00 & 0.00 & 31.25 & 0.00 & 0.00 & 0.00 \\
\hline 7 & 0.00 & 0.00 & 0.00 & 0.00 & 0.00 & 0.00 & 0.00 & 0.00 & 0.00 & 0.00 & 0.00 & 0.00 & 0.00 & 0.00 & 0.00 & 44.00 & 0.00 & 0.00 & 0.00 \\
\hline 8 & 0.00 & 0.00 & 0.00 & 0.00 & 0.00 & 0.00 & 0.00 & 0.00 & 0.00 & 0.00 & 0.00 & 0.00 & 0.00 & 0.00 & 0.00 & 0.00 & 0.00 & 0.00 & 15.63 \\
\hline 9 & 0.00 & 0.00 & 0.00 & 0.00 & 0.00 & 0.00 & 0.00 & 0.00 & 0.00 & 0.00 & 0.00 & 0.00 & 0.00 & 0.00 & 0.00 & 0.00 & 0.00 & 0.00 & 42.86 \\
\hline 10 & 0.00 & 0.00 & 0.00 & 0.00 & 0.00 & 0.00 & 0.00 & 0.00 & 0.00 & 0.00 & 0.00 & 0.00 & 0.00 & 0.00 & 0.00 & 5.00 & 0.00 & 0.00 & 0.00 \\
\hline 11 & 0.00 & 0.00 & 0.00 & 0.00 & 0.00 & 0.00 & 0.00 & 0.00 & 0.00 & 0.00 & 0.00 & 0.00 & 0.00 & 0.00 & 66.67 & 0.00 & 0.00 & 0.00 & 0.00 \\
\hline 12 & 0.00 & 0.00 & 35.08 & 0.00 & 0.00 & 0.00 & 3.24 & 1.90 & 0.00 & 0.00 & 10.72 & 0.00 & 0.00 & 0.00 & 20.62 & 0.00 & 0.00 & 0.00 & 0.00 \\
\hline 13 & 0.00 & 0.00 & 0.00 & 0.00 & 0.00 & 0.00 & 0.00 & 0.00 & 0.00 & 0.00 & 0.00 & 0.00 & 0.00 & 0.00 & 0.00 & 62.55 & 37.45 & 0.00 & 0.00 \\
\hline 14 & 0.00 & 0.00 & 0.00 & 0.00 & 0.00 & 0.00 & 0.00 & 0.00 & 0.00 & 0.00 & 0.00 & 0.00 & 0.00 & 0.00 & 0.00 & 0.00 & 40.00 & 0.00 & 0.00 \\
\hline 15 & 0.00 & 0.00 & 0.00 & 0.00 & 0.00 & 0.00 & 0.00 & 0.00 & 0.00 & 0.00 & 0.00 & 0.00 & 0.00 & 0.00 & 0.00 & 0.00 & 0.00 & 0.00 & 93.02 \\
\hline$R_{1}$ & 0.00 & 0.00 & 0.00 & 23.76 & 0.00 & 20.83 & 9.10 & 3.09 & 14.33 & 0.00 & 48.79 & 52.65 & 83.33 & 28.57 & 0.00 & 0.00 & 0.00 & 0.00 & 0.00 \\
\hline$R_{2}$ & 0.00 & 0.00 & 0.00 & 0.00 & 0.00 & 0.00 & 0.00 & 0.00 & 0.00 & 0.00 & 0.00 & 0.00 & 0.00 & 0.00 & 0.00 & 0.00 & 0.00 & 0.00 & 0.00 \\
\hline$R_{3}$ & 0.00 & 0.00 & 0.00 & 0.00 & 0.00 & 0.00 & 0.00 & 0.00 & 0.00 & 0.00 & 0.00 & 0.00 & 0.00 & 0.00 & 0.00 & 0.00 & 0.00 & 0.00 & 0.00 \\
\hline
\end{tabular}

\section{Supplementary material}

Supplementary material associated with this article can be found, in the online version, at 10.1016/j.compchemeng.2020. 107053

\section{References}

Alexander, B., Barton, G., Petrie, J., Romagnoli, J., 2000. Process synthesis and optimisation tools for environmental design: methodology and structure. Comput. Chem. Eng. 24, 1195-1200.

Almaraz, S.D.-L., Boix, M., Montastruc, L., Azzaro-Pantel, C., Liao, Z., Domenech, S., 2016. Design of a water allocation and energy network for multi-contaminant problems using multi-objective optimization. Process Saf. Environ. Prot. 103, 348-364. Process integration for sustainable resource and waste management

Aussel, D., Svensson, A.. A short state of the art on Multi-Leader-Follower Games. p. $25 \mathrm{pp}$.

Aussel, D., Svensson, A., 2019. Is pessimistic bilevel programming a special case of a mathematical program with complementarity constraints? J. Optim. Theory Appl. 181 (2), 504-520. doi:10.1007/s10957-018-01467-7.

Baumrucker, B.T., Renfro, J., Biegler, L., 2008. MPEC problem formulations and solution strategies with chemical engineering applications. Comput. Chem. Eng. 32 (12), 2903-2913.

Bezanson, J., Edelman, A., Karpinski, S., Shah, V.B., 2017. Julia: a fresh approach to numerical computing. SIAM Rev. 59 (1), 65-98.

Boix, M., Montastruc, L., Azzaro-Pantel, C., Domenech, S., 2015. Optimization methods applied to the design of Eco-industrial parks: a literature review. J. Clean. Prod. 87, 303-317.

Boix, M., Montastruc, L., Pibouleau, L., Azzaro-Pantel, C., Domenech, S., 2011. Eco industrial parks for water and heat management. Comput. Aided Chem. Eng. 29, 1175-1179.

Boix, M., Montastruc, L., Pibouleau, L., Azzaro-Pantel, C., S., D., 2012. Industrial water management by multiobjective optimization: from individual to collective solution through Eco-industrial parks. J. Clean. Prod. 22, 85-97.

Bolton, P., Dewatripont, M., 2005. Contract Theory. MIT Press, Cambridge, Mass.

Chertow, M.R., 2000. Industrial symbiosis: literature and taxonomy. Annu. Rev. Energy Env. 25 (1), 313-337. doi:10.1146/annurev.energy.25.1.313.

Dempe, S., Dutta, J., 2012. Is bilevel programming a special case of a mathematical program with complementarity constraints? Math. Program. 131 (1-2, Ser. A), 37-48. doi:10.1007/s10107-010-0342-1.

Dempe, S., Kalashnikov, V., Pérez-Valdés, G.A., Kalashnykova, N., 2015. Bilevel Programming Problems : Theory, Algorithms and Applications to Energy Networks. Springer, Heidelberg New York.

Dempe, S., Zemkoho, A., 2020. Bilevel Optimization: Advances and Next Challenges. Springer, Springer, New York.

Emmerich, M.T.M., Deutz, A.H., 2018. A tutorial on multiobjective optimization: fundamentals and evolutionary methods. Nat. Comput. 17, 585-609. doi:10.1007| s11047-018-9685-y.
Facchinei, F., Kanzow, C., 2010. Generalized Nash equilibrium problems. Ann. Oper Res. 175, 177-211. doi:10.1007/s10479-009-0653-x.

Frosch, A., Gallopoulos, N.E., 1989. Strategies for manufacturing. Sci. Am. 261 144-152.

LLC Gurobi Optimization, 2020. Gurobi optimizer reference manual.

Hu, M., Fukushima, M., 2015. Multi-leader-follower games: models, methods and applications. J. Oper. Res. Soc. Jpn. 58 (1), 1-23. doi:10.15807/jorsj.58.1.

chiishi, T. 1983. Game Theory for Economic Analysis. Economic Theory, Econometrics, and Mathematical Economics. Academic Press, Inc. [Harcourt Brace Jovanovich, Publishers], New York.

Jackson, M. O., 2014. Mechanism Theory. Available at SSRN: https://ssrn.com/ abstract=2542983or https://doi.org/10.2139/ssrn.2542983.

Kastner, C., Lau, R., Kraft, M., 2015. Quantitative tools for cultivating symbiosis in industrial parks; a literature review. Appl. Energy 155, 599-612.

Luo, Z.-Q., J.-S., P., Ralph, D., 1996. Mathematical Programs with Equilibrium Constraints. Cambridge University Press, Cambridge New York.

McCain, R., 2010. Game Theory: A Nontechnical Introduction to the Analysis of Strategy. World Scientific, Hackensack, NJ.

Montastruc, L., Boix, M., Pibouleau, L., Azzaro-Pantel, C., Domenech, S., 2013. On the flexibility of an Eco-industrial park (EIP) for managing industrial water. J. Clean. Prod. 43, 1-11. doi:10.1016/j.jclepro.2012.12.039.

Neves, A., Godina, R., Azevedo, S.G., Matias, J.C., 2020. A comprehensive review of industrial symbiosis. J. Clean. Prod. 247, 119113.

Nisan, N., Roughgarden, T., Tardos, E., Vazirani, V.V., 2007. Algorithmic Game Theory. Cambridge University Press, Cambridge New York.

Olesen, S., Polley, G., 1996. Dealing with plant geography and piping constraints in water network design. Process. Saf. Environ. Prot. 74, 273-276.

Pang, J.-S., Fukushima, M., 2005. Quasi-variational inequalities, generalized Nash equilibria, and multi-leader-follower games. Comput. Manag. Sci. 2 (1), 21-56. doi:10.1007/s10287-004-0010-0.

UNEP (United Nations Environmental Programme), 2000. Global environmental outlook 2000. Earthscan, London.

Ramos, M., Boix, M., Aussel, D., Montastruc, L., Domenech, S., 2016. Water integration in Eco-industrial parks using a multi-leader-follower approach. Comput. Chem. Eng. 87, 190-207.

Ramos, M., Rocafull, M., Boix, M., Aussel, D., L., M., Domenech, S., 2018. Utility network optimization in Eco-industrial parks by a multi-leader-follower game methodology. Comput. Chem. Eng. 112, 132-153.

Ramos, M., Rocafull, M., Boix, M., Aussel, D., Montastruc, L., Domenech, S., 2018 Utility network optimization in Eco-industrial parks by a multi-leader follower game methodology. Comput. Chem. Eng. 112, 132-153. doi:10.1016/j. compchemeng.2018.01.024.

Salanié, B., 2005. The Economics of Contracts: A primer. MIT Press, Cambridge, Mass.

Scientific, U.U.N.E., Organization), C., 2009. The united nations world water development. Water in a Changing World; Report 3.

Tseveendorj, I., 2013. Mathematical programs with equilibrium constraints: a brief survey of methods and optimality conditions. In: Optimization, simulation, and control. In: Springer Optim. Appl., 76. Springer, New York, pp. 49-61. 